\title{
Pharmacokinetics of Anthraquinones from Medicinal Plants
}

\author{
Dongpeng Wang ${ }^{1,2 \#}$, Xian-He Wang ${ }^{1 \#}$, Xiongjie Yu ${ }^{1}$, Fengjun Cao ${ }^{1}$, Xiaojun Cai ${ }^{1}$, Ping Chen ${ }^{1}$, \\ Minglun $\mathrm{Li}^{3}$, Yibin Feng ${ }^{4}$, Hongliang $\mathrm{Li}^{1,2 *}$ and Xuanbin Wang ${ }^{1,2,3 *}$ \\ ${ }^{1}$ Laboratory of Chinese Herbal Pharmacology, Oncology Center, Renmin Hospital, Hubei University of Medicine, Shiyan, China, \\ ${ }^{2}$ Biomedical Research Institute, Hubei Key Laboratory of Wudang Local Chinese Medicine Research and School of Pharmacy, \\ Hubei University of Medicine, Shiyan, China, ${ }^{3}$ Department of Radiation Oncology, University Hospital, LMU Munich, Munich, \\ Germany, ${ }^{4}$ School of Chinese Medicine, The University of Hong Kong, Hong Kong, China
}

\section{OPEN ACCESS}

Edited by:

Haitao Lu,

Shanghai Jiao Tong University, China

Reviewed by:

Hongtao Liu,

Hubei University of Chinese Medicine,

China

Ying-yuan Lu,

State Key Laboratory of Natural and Biomimetic Drugs, Peking University,

China

*Correspondence:

Xuanbin Wang

wangxb@hbmu.edu.cn Hongliang $L$

hongliangli@hbmu.edu.cn

\#These authors have contributed equally to this work

Specialty section:

This article was submitted to

Ethnopharmacology,

a section of the journal

Frontiers in Pharmacology

Received: 08 December 2020

Accepted: 03 February 2021

Published: 15 April 2021

Citation:

Wang $D$, Wang $X-H, Y U X, C a O F$, Cai $X$, Chen P, Li M, Feng Y, Li H and

Wang $X$ (2021) Pharmacokinetics of Anthraquinones from Medicinal Plants.

Front. Pharmacol. 12:638993.

doi: 10.3389/fphar.2021.638993
Anthraquinones are bioactive natural products, some of which are active components in medicinal medicines, especially Chinese medicines. These compounds exert actions including purgation, anti-inflammation, immunoregulation, antihyperlipidemia, and anticancer effects. This study aimed to review the pharmacokinetics (PKs) of anthraquinones, which are importantly associated with their pharmacological and toxicological effects. Anthraquinones are absorbed mainly in intestines. The absorption rates of free anthraquinones are faster than those of their conjugated glycosides because of the higher liposolubility. A fluctuation in blood concentration and two absorption peaks of anthraquinones may result from the hepato-intestinal circulation, reabsorption, and transformation. Anthraquinones are widely distributed throughout the body, mainly in blood-flow rich organs and tissues, such as blood, intestines, stomach, liver, lung, kidney, and fat. The metabolic pathways of anthraquinones are hydrolysis, glycuronidation, sulfation, methylation/demethylation, hydroxylation/dehydroxylation, oxidation/reduction (hydrogenation), acetylation and esterification by intestinal flora and liver metabolic enzymes, among which hydrolysis, glycuronidation and sulfation are dominant. Of note, anthraquinones can be transformed into each other. The main excretion routes for anthraquinones are the kidney, recta, and gallbladder. Conclusion: Some anthraquinones and their glycosides, such as aloe-emodin, chrysophanol, emodin, physcion, rhein and sennosides, have attracted the most PK research interest due to their more biological activities and/or detectability. Anthraquinones are mainly absorbed in the intestines and are mostly distributed in blood flow-rich tissues and organs. Transformation into another anthraquinone may increase the blood concentration of the latter, leading to an increased pharmacological and/or toxicological effect. Drugdrug interactions influencing PK may provide insights into drug compatibility theory to enhance or reduce pharmacological/toxicological effects in Chinese medicine formulae and deserve deep investigation.

Keywords: anthraquinones, pharmacokinetics, Chinese medicines, natural products, medicinal plant 


\section{INTRODUCTION}

Anthraquinones naturally exist in plant families, such as, Polygonaceae, Leguminosae, Rubiaceae (Chen et al., 2020), Rhamnaceae, Scrophulariaceae, Liliaceae, Verbenaceae and Valerianaceae (Zhao et al., 2011), e.g., Rheum palmatum L., Rheum tanguticum Maxim, ex Balf., Rheum officinale Baill., Cassia obtusifolia L., Cassia tora L., Verbena officinalis L., Polygonum multiflorum Thunb., Aloe barbadmsis Miller., Aloe ferox Miller, Rubia cordifolia L., Cassia angustifolia Vahl, Cassia acutifolia Delile, and Morinda angustifolia Roxb (Chen et al., 2020). Anthraquinones are also found in the secondary metabolites of lower-order plants, such as, lichens (Solárová et al., 2020). Some plants have been used for Chinese medicines (Yang et al., 2016; Li H. et al., 2018; Yang et al., 2018) (Figures 1A-F).

Pharmacological studies have shown that anthraquinones exert purgative (Gong et al., 2015), anti-inflammatory (Li D. et al., 2013), immunoregulation (Abu et al., 2018), antihyperlipidemia (Wang et al., 2014), and anticancer effects (Lin et al., 2015; Cui et al., 2016; Yang N. et al., 2019). Thus, pharmacokinetics (PKs) has attracted increasing attention and in-depth research for scholars, especially in the field of Chinese medicines.

Anthraquinones are structurally divided into two classes, mononuclear and dinuclear. Their names and CAS numbers are listed in Supplementary Table S1. The parent rings of anthraquinones are illustrated in Figure 1G.

Thanks to advanced technologies and methodologies, the pharmacological and/or toxicological effects of anthraquinones have been gradually uncovered. However, there has been no overall review of their PKs untill now, which are closely associated with their bioactions. Thus, this study summarized the PKs of anthraquinones, aiming to provide basic knowledge for further research on the pharmacological and toxicological effects and mechanisms of anthraquinones.

\section{ABSORPTION}

\section{Absorption Sites and Rate}

The absorption of anthraquinones depends on their physical and chemical properties, especially quinone structure and liposolubility under the normal conditions. The dominant absorption sites for anthraquinones are the intestines rather than the stomach (Wang J. et al., 2011; Liu X. et al., 2011; Wang P. et al., 2011), although emodin is absorbed more quickly in the stomach than in the intestines (Kong et al., 2011). This may result from anthraquinones having more retention time in the intestines than in the stomach (Kong et al., 2011).

Regarding the intestines, the accumulated absorption rates of total anthraquinones in the small intestines and colons of male SD rats at $2 \mathrm{~h}$ are 66.99 and $23.54 \%$, respectively (Liu X. et al., 2011). Anthraquinones can easily enter small intestinal villi epithelial cells through passive diffusion (Li et al., 2012). This can be calculated via their absorption rate constant $\left(K_{\mathrm{a}}\right)$ and apparent absorption coefficient $\left(P_{\text {app }}\right)$ (Table 1) (Figure 1H). A larger $K_{\mathrm{a}}$ means a shorter $\mathrm{T}_{\max }$, i.e., faster drug absorption. A larger $P_{\text {app }}$ means a larger area under the curve (AUC). Actually, the $P_{\text {app }}$ of anthraquinones is the greatest in the duodenum and then decreased in the jejunum and are minimum in the ileum (Qiu et al., 2011; Wang J. et al., 2011; Wang P. et al., 2011). However, the $K_{\mathrm{a}}$ and $P_{\mathrm{app}}$ of anthraquinones increased in the colon than that in the ileum (Figure 1H). This may be associated with the weak acidity of anthraquinones and the $\mathrm{pH}$ conditions in the intestines. Since the upper small intestines are a weakly acidic environment (Wang J. et al., 2011; Wang P. et al., 2011), given that most anthraquinones are weakly acidic, this may lead to lower ionization and higher liposolubility of anthraquinones. In contrast, with a higher $\mathrm{pH}$ value, the ileum is an alkaline environment $(\mathrm{pH}=7-8)$, where the ionization degree of anthraquinones is increased leading to little anthraquinone absorption. However, compaired with the ileum, the $K_{\mathrm{a}}$ and $P_{\text {app }}$ of anthraquinones increase in colons because the acidity increases slightly and the retention time is prolonged (Table 1).

Generally, anthraquinones are absorbed with over a wide range in vivo. This may be due to differences in drug dosages, detection instruments, and protocols. As described in Table 2, the greater the body weight of the subjects is, the greater the $C_{\max }$ and AUC are. Among anthraquinones, rhein has the lowest $\mathrm{T}_{\max }$, and the highest $C_{\max }$ and AUC in dogs (Zhu et al., 2006) (Table 2).

\section{Affecting Factors Physiological Conditions}

Experimental Animal Species The absolute bioavailability (F) of rhein in beagle dogs is higher than that in rats ( 49.7 vs. $23.8 \%, p<$ 0.01) (Zhang et al., 2010) (Table 1).

Sex The AUC values of emodin (Liu W. et al., 2011) and aloeemodin (Yang et al., 2010) in male rats are higher than those in female rats. In contrast, the AUC of rhein in healthy women is higher than that in men. Furthermore, the $T_{\max }$ of rhein is shorter in women than that in men (Zhu et al., 2006), indicating a faster absorption of rhein in female (Yang et al., 2010). These findings may result from the difference in the body fat ratio between females and males (Zhu et al., 2006).

Hepato-Intestinal Circulation and Reabsorbing The blood levels of aloe-emodin, chrysophanol, emodin, chrysoobtusin, physcion-8-O- $\beta$-D-glucoside, chrysophanol-8-O- $\beta$-D-glucoside, obtusin, aurantio-obtusin, obtusifolin, physcion and rhein fluctuate dramatically due to the hepato-intestinal circulation (and glycoside hydrolysis in the intestines) (Ullah et al., 2018; Yang B. et al., 2019). Another factor is that anthraquinones are rapidly distributed to other organs and re-absorbed into the blood. Thus, aurantio-obtusin, obtusin, chrysoobtusin, emodin, chrysophanol, rhein and aloe-emodin form second absorption peaks. For example, the second absorption peaks for emodin from different studies range from approximately $3-10 \mathrm{~h}$ (Wu W. J. et al., 2017; Wang L. et al., 2017; Ullah et al., 2018; Yang B. et al., 2019; Zhang et al., 2019).

Food Compared with the fasted rats, the $C_{\max }$ and AUC of rhein and emodin increase in the fed group (Gong et al., 2011). However, the mechanism is currently unknown. 


\section{Disorders}

The AUC values of rhein, aloe-emodin, chrysophanol, emodin and physcion increase significantly in the rats with microcirculation disturbance compaired with the normal group (Zhu et al., 2017). The $C_{\max }$ and $T_{\max }$ of chrysophanol are increased in acute pancreatitis in dogs compaired with the normal group (Yang et al., 2012). Conversely, rhein had a lower AUC in liver-injured male rats. The potential mechanism may result from changes in the expression and activation of metabolic enzymes in the injured liver (Zhang et al., 2015). For constipated rats, oral administration of rhubarb extract (anthraquinone-rich containing plant) resulted in a the $C_{\max }$ and AUC of emodin that were approximately ten times those of normal rats, while the AUC values for aloe-emodin and rhein were decreased. The mechanisms may be attributed to the direct action of aloeemodin and rhein on intestinal cell membranes and the indirect action of emodin on bowel movement through adjustment by the nervous system (Gong et al., 2015). This may synergistically enhance the purgative effect on constipation.

\section{Drug-Drug Interactions}

Drug-drug interactions always alter the single herb pharmacological effects. Since natural products especially Chinese medicines are always used as formulae that consist of two or more herbs, they play a critical role in investigating the influencing factors of drug-drug interactions in PKs. Generally, the combination of anthraquinones with other drugs has three types, pure compounds of anthraquinones, anthraquinonecontaining single herbs (including their extracts and fractions), and anthraquinone-containing herbs in formulae.

Anthraquinone-containing single herbs combined with other drugs or single-herbs (herb pairs) are a basic building block for Chinese medicine use. For example, a rhubarb-gardenia herb pair consisting of Rhei Radix et Rhizoma (Dahuang containing anthraquinones) (Figures 1A,D) and Gardeniae Flos (Zhizihua, containing genipin) is used for treating cholestasis diseases. A study showed that Gardeniae Flos increased the $C_{\max }$ and AUC of aloe-emodin, chrysophanol, emodin and rhein in rats, indicating a synergistic effect of the rhubarb-gardenia herb pair on hepatoprotection (Dong et al., 2015).

Compared with pure compounds and single herbs, interactions between herbs in a formula are the most common to assess drug compatibility for traditional Chinese medicines.

Da-Cheng-Qi decoction (DCQD), a classic formula including Rhei Radix et Rhizoma ("monarch" herb), Magnoliae Officinalis Cortex (Houpo), Aurantii Fructus Immaturus (Zhishi), and Natrii Sulfas (Mangxiao, $\mathrm{Na}_{2} \mathrm{SO}_{4} \cdot 10 \mathrm{H}_{2} \mathrm{O}$ ), has been used for treating acute pancreatitis and intestinal obstruction. Combining DCQD with ranitidine (an $\mathrm{H} 2$ receptor inhibitor) is a Chinese-Western integrative strategy for such diseases. Thus, it is necessary to investigate the drug-drug interactions between ranitidine and DCQD. Ren et al. reported that ranitidine reduces the $C_{\max }$ and AUC of rhein in DCQD. Therefore, the bioavailability of DCQD may be decreased, indicating the dosage of DCQD should be increase when combined with ranitidine. This may result from ranitidine changing gastrointestinal motility and inhibiting the absorption of rhein. (Ren et al., 2009).

San-Huang tablets, consisting of Rhei Radix et Rhizoma, extracts of Scutellariae Radix (Huangqin) and berberine hydrochloride, are used for multiple diseases, such as constipation, inflammation, pathogenic microbes, and spasm. Rhei Radix et Rhizoma is the main component for constipation because of its active compound, emodin. Studies have shown that Scutellariae Radix and/or berberine hydrochloride increased the AUC and $C_{\max }$ of emodin, indicating a potentiation role of Scutellariae Radix and/or berberine hydrochloride in the efficacy of emodin (Zhou et al., 2010). Moreover, Xin et al. reported that San-Huang-Xie-Xin decoction (SHXXD), including Rhei Radix et Rhizoma, Scutellariae Radix and Coptidis Rhizoma (containing berberine), increases the $C_{\max }$ and AUC of rhein compared with a single herb of Rhei Radix et Rhizoma (Xin et al., 2009). The mechanisms may be due to the inhibited glucuronidation activity of UDP-glucuronyltransferases (UGTs) by other ingredients in SHXXD, leading to the increased bioavailability of rhein (Hou et al., 2014).

Dahuang-mudan decoction (DMD) consists of Rhei Radix et Rhizoma, Moutan Cortex (Mudanpi), Persicae Semen (Taoren), Benincasae Semen (Dongguaren), and Natrii Sulfas. DMD has been used for treating intestinal carbuncles for approximately 1,700 years since the Han Dynasty. Pharmacological effects on appendicitis, inflammatory bowel disease, pelvic inflammatory disease and acute pancreatitis have been found with the identification of active compounds, emodin, aloe-emodin, rhein, paeoniflorin and amygdalin. Nong et al. reported that Natrii Sulfas decreases the $C_{\max }$ and AUC of emodin and rhein while increasing the absorption of aloe-emodin, indicating novel insight into the role of Natrii Sulfas in DMD in addition to a stool softener treatment of archenteric inflammatory disease (Zhang Y. X. et al., 2013; Nong et al., 2019).

Tao-He-Cheng-Qi-Tang (THCQT), including Persicae Semen (Taoren), Rhei Radix et Rhizoma, Natrii Sulfas, Cinnamomi Ramulus (Guizhi), and Glycyrrhizae Radix et Rhizoma (Gancao), has been used to treat platelet aggregation, hyperlipidemia, diabetes, inflammation, and related conditions. Xie et al. reported that compared with the oral administration of Rhei Radix et Rhizoma alone, the $C_{\max }$ and AUC of rhein in THCQT increased in rabbits. However, the mechanisms for the alternation of rhein absorption are unknown (Xie et al., 2005).

An eight-herb formula, Niu-Huang-Jie-Du tablets (NHJDT), including Bovis Calculus (Niuhuang), Rhei Radix et Rhizoma, Realgar $\left(\mathrm{As}_{2} \mathrm{~S}_{2}\right.$, Xionghuang), Gypsum Fibrosum $\left(\mathrm{CaSO}_{4} \cdot 2 \mathrm{H}_{2} \mathrm{O}\right.$, Shigao), Platycodonis Radix (Jiegeng), and Borneolum Syntheticum (D-borneoland, Bingpian), exerts heat clearance and detoxification in Chinese medicine. Compaired with oral adminstraton of Rhei Radix et Rhizoma alone in rats, the AUC and $C_{\max }$ of rhein increased in NHJDT, while the $T_{\max }$ of the chrysophanol isomer decreased. The mechanism requires further study (Liu Y. et al., 2018). 

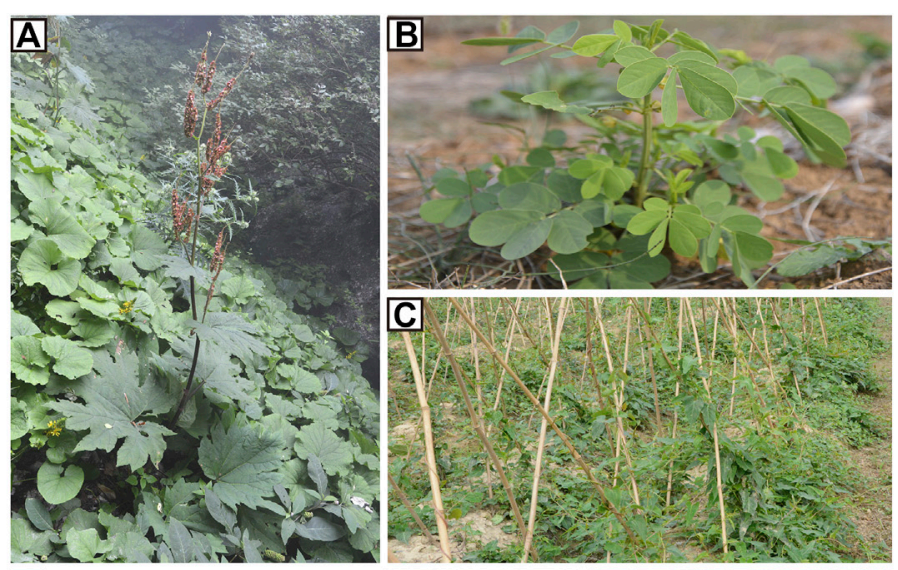

G
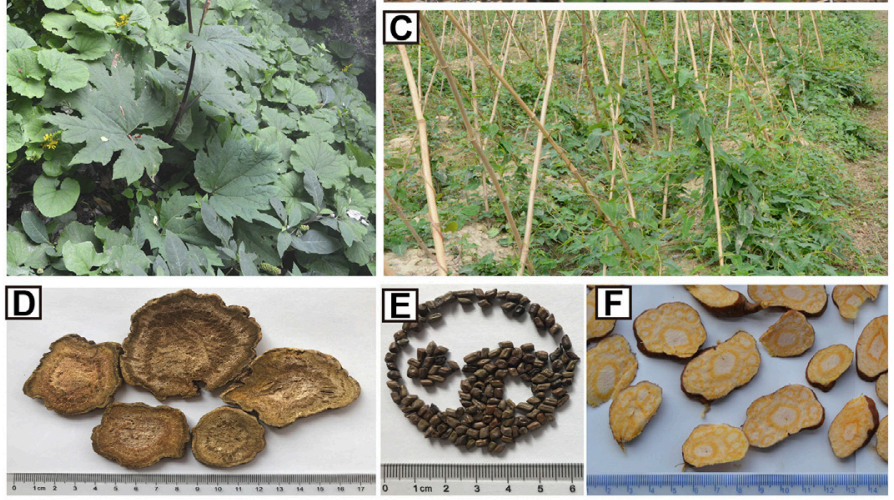<smiles>[R2]c1cc(O)c2c(c1)C(=O)c1c(OC)cccc1C(c1cccc(OCCl)c1)c1cc([R2])cc(O)c1C2</smiles><smiles>[R2]c1cc(O)c2c(c1)C(=O)c1cc([R2])cc(O)c1C2=O</smiles>

anthraquinones

\section{Dinuclear anthraquinones}
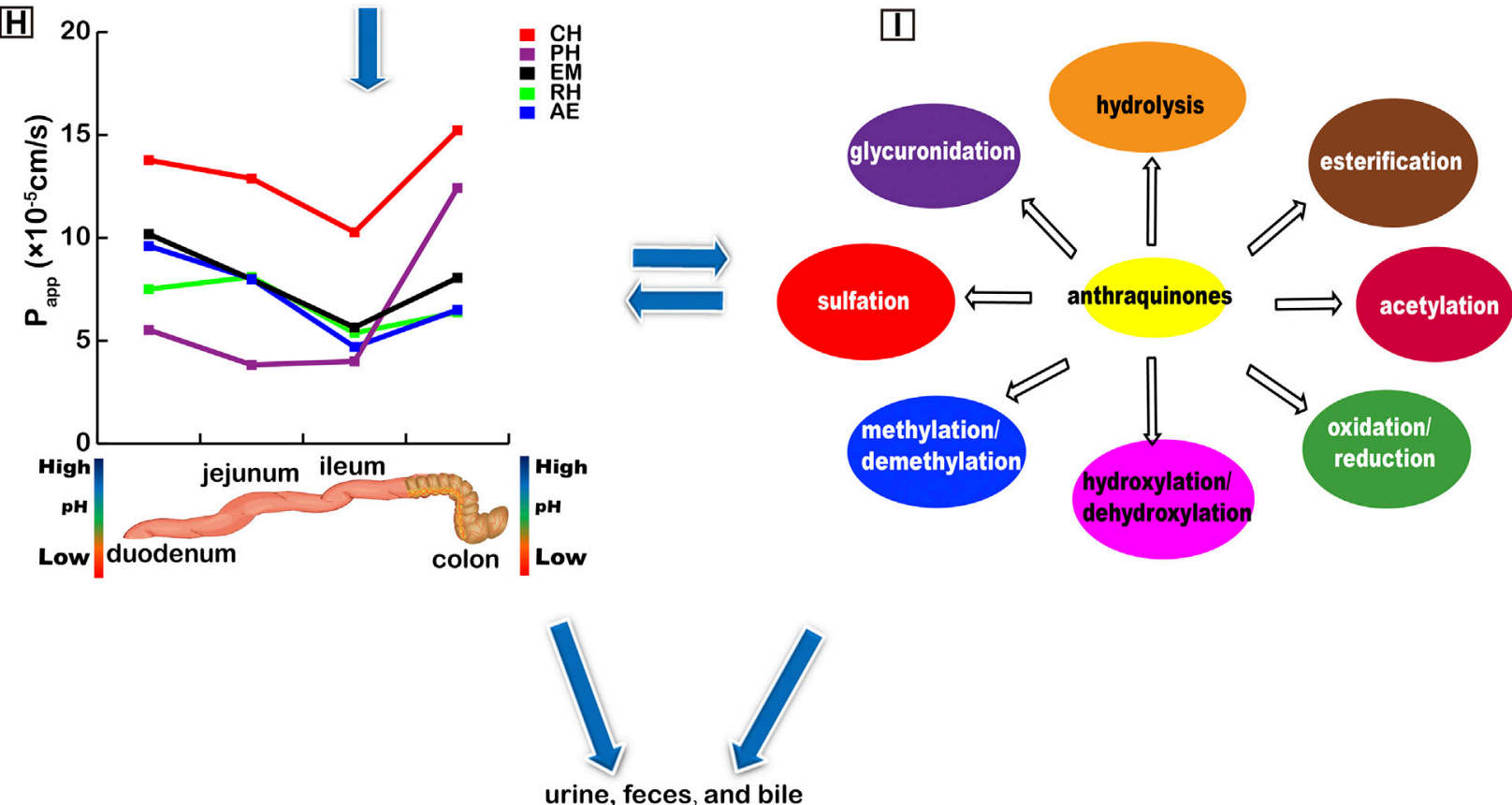

FIGURE 1 | Pharmacokinetics (PK) of anthraquinones. (A) Rheum officinale Baill. (B) Cassia tora L. (C) Polygonum multiflorum Thunb. (D) Rhei Radix et Rhizoma. (E) Cassiae Semen. (F) Polygoni Multiflori Radix. (G) structures of anthraquinones and dinuclear anthraquinone glycosides. R1 and R2 represent different groups including glucoses. (H) absorption of anthraquinones. $P_{\text {app: }}$ apparent absorption coefficient. CH: chrysophanol; PH: physcion; EM: emodin; RH: rhein; AE: aleo-emodin. (I) metabolism of anthraquinones.

\section{DISTRIBUTION}

\section{Tissues and Organs}

Since the bioavailability of anthraquinones is low, to date, only a few distribution studies of the anthraquinones aloe-emodin, chrysophanol, emodin, rhein, and physcion have been reported, as listed in Table 3 . These anthraquinones are widely distributed and are more abundant in blood-rich tissues and organs, such as the intestines, stomach, plasma, lung, liver, heart, and kidney. More intestine and stomach distribution may facilitate anthraquinone treatment of digestive gut disorders. They are also detected in fat, possibly due to their good liposolubility. However, few anthraquinones have been discovered in the brain since they have difficulty passing through the blood-brain barrier (Ding et al., 2003; Shia et al., 2011b; Tan et al., 2013; Chen et al., 2014; Du et al., 
TABLE 1 $K_{\mathrm{a}}$ and $P_{\text {app }}$ values of some anthraquinone compounds absorbed in different intestines and colons of rats.

\begin{tabular}{|c|c|c|c|c|c|c|c|c|c|}
\hline & \multicolumn{2}{|c|}{ Duodenum } & \multicolumn{2}{|c|}{ Jejunum } & \multicolumn{2}{|c|}{ lleum } & \multicolumn{2}{|c|}{ Colons } & \multirow[t]{2}{*}{ References } \\
\hline & $\begin{array}{c}K_{\mathrm{am}}\left(\times 10^{-}\right. \\
4 / \mathrm{s})\end{array}$ & $\begin{array}{c}P_{\text {app }}\left(\times 10^{-}\right. \\
5 \mathrm{~cm} / \mathrm{s})\end{array}$ & $\begin{array}{c}K_{\mathrm{a}}\left(\times 10^{-}\right. \\
4 / \mathrm{s})\end{array}$ & $\begin{array}{c}P_{\text {app }}\left(\times 10^{-}\right. \\
5 \mathrm{~cm} / \mathrm{s})\end{array}$ & $\begin{array}{c}K_{\mathrm{a}}\left(\times 10^{-}\right. \\
4 / \mathrm{s})\end{array}$ & $\begin{array}{c}P_{\text {app }}\left(\times 10^{-}\right. \\
5 \mathrm{~cm} / \mathrm{s})\end{array}$ & $\begin{array}{c}K_{\mathrm{a}}\left(\times 10^{-}\right. \\
4 / \mathrm{s})\end{array}$ & $\begin{array}{c}P_{\text {app }}\left(\times 10^{-}\right. \\
\left.{ }^{5} \mathrm{~cm} / \mathrm{s}\right)\end{array}$ & \\
\hline$A E$ & $5.43-16.07$ & $7.65-10.68$ & $4.88-13.03$ & $6.29-9.83$ & $2.23-8.63$ & $3.45-5.90$ & $3.88-12.17$ & $5.12-7.9$ & (Wang et al., 2011a; Wang et al., \\
\hline $\mathrm{CH}$ & 19.02 & 13.77 & 15.15 & 12.88 & 10.80 & 10.27 & 18.17 & 15.22 & 2011b; Qiu et al., 2011) \\
\hline EM & 15.55 & 10.18 & 11.45 & 7.98 & 8.38 & 5.65 & 12.45 & 8.05 & \\
\hline $\mathrm{PH}$ & 10.08 & 5.53 & 6.38 & 3.83 & 6.22 & 4.00 & 16.12 & 12.42 & \\
\hline $\mathrm{RH}$ & $6.96-10.68$ & $6.15-8.91$ & $5.70-11.13$ & $7.95-8.22$ & $4.79-6.27$ & $4.17-6.59$ & $5.18-6.55$ & $3.85-8.92$ & \\
\hline
\end{tabular}

$\mathrm{K}_{a}$ : absorption rate constant; $\mathrm{P}_{\text {app: }}$ apparent absorption coefficient; $A$ E: aloe-emodin; $C H$ : chrysophanol; EM: emodin; $P H$ : physcion; RH: rhein. The number of male and female rats in the studies was equal.

TABLE 2 | The pharmacokinetic parameters of anthraquinones in rats and dogs.

\begin{tabular}{|c|c|c|c|c|c|c|c|}
\hline \multirow{2}{*}{$\begin{array}{l}\text { Pharmacokinetic } \\
\text { parameters }\end{array}$} & \multicolumn{2}{|c|}{$C_{\max }(\mu \mathrm{g} / \mathrm{ml})$} & \multicolumn{2}{|c|}{$T_{\max }(\mathrm{h})$} & \multicolumn{2}{|c|}{ AUC0- $\infty$ mg/(L.h) } & \multirow[t]{2}{*}{ References } \\
\hline & Rats & Dogs & Rats & Dogs & Rats & Dogs & \\
\hline Aloe-emodin & $0.004-124.40$ & $0.03-0.45$ & $0.20-11.33$ & $0.75-1.55$ & $0.008-4.67$ & $0.42-1.61$ & $\begin{array}{l}\text { (Yang et al., 2012; Zhang et al., 2013b; Feng et al., } \\
\text { 2013; Feng et al., 2014; Jiang et al., 2015; Yang } \\
\text { et al., 2019a; Cheng et al., 2020) }\end{array}$ \\
\hline Alizarin & 0.25 & - & 0.98 & - & 1.64 & - & (Gao et al., 2018) \\
\hline Aurantio-obtusin & $0.17-1,135.80$ & - & $0.08-0.53$ & - & $0.99-5.90$ & - & $\begin{array}{l}\text { (Zhang et al., 2014; Yang et al., 2015; Guo et al., } \\
\text { 2017; Yang et al., 2019a) }\end{array}$ \\
\hline Chrysophanol & $0.001-3,142.80$ & $0.04-0.30$ & $0.25-9.28$ & $1.00-2.00$ & $0.01-37.05$ & $0.54-0.83$ & $\begin{array}{l}\text { (Yang et al., 2012; Feng et al., 2014; Zhu et al., 2017; } \\
\text { Ullah et al., 2018; Yang et al., 2019a; Cheng et al., } \\
\text { 2020) }\end{array}$ \\
\hline $\begin{array}{l}\text { Chrysophanol-8-O- } \beta-\mathrm{D}- \\
\text { glycoside }\end{array}$ & 0.03 & - & 2.00 & - & 0.158 & - & (Ullah et al., 2018) \\
\hline Chryso-obtusin & $0.05-894.1$ & - & $0.08-3.64$ & - & $0.27-3.58$ & - & (Zhang et al., 2014; Yang et al., 2019a) \\
\hline Citreorosein & 0.149 & - & 0.19 & - & 0.134 & - & (Cheng et al., 2020) \\
\hline Emodin & $0.001-348.4$ & $0.27-0.48$ & $0.10-8.94$ & $0.75-1.42$ & $0.004-39.6$ & $1.38-4.05$ & $\begin{array}{l}\text { (Song et al., 2009b; Yang et al., 2012; Feng et al., } \\
\text { 2014; Zhu et al., 2014; Jiang et al., 2015; Zhu et al., } \\
\text { 2017; Yang et al., 2019a; }\end{array}$ \\
\hline $\begin{array}{l}\text { Emodin-8-O- } \beta-D- \\
\text { glycoside }\end{array}$ & $0.02-0.10$ & - & $0.28-0.29$ & - & $0.014-0.084$ & - & (Zhang et al., 2018b; Cheng et al., 2020) \\
\hline Munjistin & $0.03-0.74$ & - & $1.61-1.93$ & - & $0.14-3.99$ & & (Gao et al., 2016; Gao et al., 2018) \\
\hline Obtusifolin & $0.10-1,535.5$ & - & $0.13-3.94$ & - & $0.24-18.17$ & - & $\begin{array}{l}\text { (Yang et al., 2015; Guo et al., 2017; Yang et al., } \\
\text { 2019a) }\end{array}$ \\
\hline Obtusin & $0.12-802.0$ & - & $0.33-1.13$ & - & $0.36-7.07$ & - & (Zhang et al., 2014; Yang et al., 2019a) \\
\hline Physcion & $0.03-0.49$ & 0.03 & $0.17-10.4$ & 2.00 & $0.07-3.29$ & 0.48 & $\begin{array}{l}\text { (Feng et al., 2013; Feng et al., 2014; Huang et al., } \\
\text { 2014; Yang et al., 2015; Feng et al., 2017; Zhu et al., } \\
\text { 2017) }\end{array}$ \\
\hline $\begin{array}{l}\text { Physcion-8-O- } \beta-D- \\
\text { glycoside }\end{array}$ & $0.019-0.021$ & - & $0.26-0.75$ & - & 0.084 & - & (Ullah et al., 2018; Cheng et al., 2020) \\
\hline Purpurin & $0.07-0.21$ & - & $1.61-1.64$ & - & $0.24-1.55$ & - & (Gao et al., 2016; Gao et al., 2018) \\
\hline Questinol & 0.001 & - & 4.38 & - & 0.017 & - & (Cheng et al., 2020) \\
\hline Questin & $0.028-0.056$ & - & $0.17-0.23$ & - & $0.22-0.26$ & - & (Guo et al., 2017) \\
\hline Rhein & $0.001-134.0$ & $1.44-3.39$ & $0.08-12.00$ & $0.71-1.50$ & $0.002-63.14$ & $4.24-35.15$ & $\begin{array}{l}\text { (Yang et al., 2012; Feng et al., 2014; Jiang et al., } \\
\text { 2015; Li et al., 2017b; Yang et al., 2019a; Cheng } \\
\text { et al., 2020) }\end{array}$ \\
\hline Xanthopurpurin & 0.06 & - & 1.3 & - & 0.34 & & (Han et al., 2020) \\
\hline 1-desmethylobtusin & 0.11 & - & 0.5 & - & 0.54 & - & (Zhang et al., 2014) \\
\hline
\end{tabular}

$\mathrm{C}_{\text {max }}$ : peak concentration; $\mathrm{T}_{\text {max }}$ : peak time; AUC: area under the curve.

2014), although chrysophanol easily enters the brain when it is prepared in liposomes (Zhu et al., 2012).

\section{Affecting Factors}

\section{Physiological Condition}

Sex After oral administration of $4.5 \mathrm{mg} / \mathrm{kg}$ of ${ }^{14} \mathrm{C}$-aloe-emodin to rats, the concentration of aloe-emodin in rat ovaries is higher than that in testes (Lang, 1993). The amounts of emodin and rhein in the liver of female rats are greater than those in male rats (Chen et al. 2017). The different distribution between males and females suggests that sex should be taken into consideration before clinical drug use.

\section{Disorders}

The distribution of anthraquinones in tissues and organs is associated with therapeutic target sites, effects and storage. More tissue distribution may involve stronger efficacy on tissues and organs. 
TABLE 3 | Distribution of anthraquinones in various tissues and organs.

\begin{tabular}{|c|c|c|c|c|c|}
\hline Components & $\begin{array}{l}\text { Species/ } \\
\text { biomatrix }\end{array}$ & $\begin{array}{l}\text { Administration } \\
\text { routines }\end{array}$ & Administration dosage & Distribution & References \\
\hline Aloe-emodin & KM mouse & i.g. & 300 mg/kg (rhubarb extract) & $\begin{array}{l}\text { Intestines, stomach, kidney, lung, muscle, liver, heart, } \\
\text { fat, brain, plasma, spleen }\end{array}$ & $\begin{array}{l}\text { (Wang et al., } \\
\text { 2020) }\end{array}$ \\
\hline Aloe-emodin & $\mathrm{KM}$ mouse & i.g. & $\begin{array}{l}52.2 \mathrm{mg} / \mathrm{kg}, 26.1 \mathrm{mg} / \mathrm{kg} \\
13.05 \mathrm{mg} / \mathrm{kg}\end{array}$ & $\begin{array}{l}\text { Intestines, heart, lung, liver, kidney, brain, stomach, } \\
\text { spleen, muscle, fat, plasma }\end{array}$ & $\begin{array}{l}\text { (Li and Feng, } \\
\text { 2018) }\end{array}$ \\
\hline Chrysophanol & KM mouse & i.g. & 300 mg/kg (rhubarb extract) & $\begin{array}{l}\text { Stomach, intestines, liver, spleen, kidney, fat, lung, } \\
\text { plasma, muscle, heart, brain }\end{array}$ & $\begin{array}{l}\text { (Wang et al., } \\
\text { 2020) }\end{array}$ \\
\hline Chrysophanol & $\begin{array}{l}\text { New Zealand } \\
\text { rabbits }\end{array}$ & i.v. & $15 \mathrm{mg} / \mathrm{kg}$ & Heart, lung, liver, kidney, brain & $\begin{array}{l}\text { (Tan et al., } \\
\text { 2013) }\end{array}$ \\
\hline Chrysophanol & $\mathrm{SD}$ rats & i.g. & $15 \mathrm{mg} / \mathrm{kg}$ & Heart, kidney, spleen, liver, lung, brain & $\begin{array}{l}\text { (Chen et al., } \\
\text { 2014) }\end{array}$ \\
\hline Chrysophanol & KM mouse & i.v. & $10 \mathrm{mg} / \mathrm{kg}$ & Blood, heart, kidney, spleen, liver, lung, brain & $\begin{array}{l}\text { (Zhu et al., } \\
\text { 2012) }\end{array}$ \\
\hline Emodin & $\mathrm{KM}$ mouse & i.v. & $(5.45 \mu \mathrm{g}, 13.7 \mathrm{nmol}) 0.1 \mathrm{ml}$ & $\begin{array}{l}\text { Blood, lung, kidney, stomach, thyroid, liver, bone, small } \\
\text { intestines, skin, heart, spleen, mucle, brain }\end{array}$ & (Du et al., 2014) \\
\hline Emodin & KM mouse & i.g. & 300 mg/kg (rhubarb extract) & $\begin{array}{l}\text { Stomach, intestines, liver, kidney, lung, spleen, plasma, } \\
\text { fat, heart, muscle, brain }\end{array}$ & $\begin{array}{l}\text { (Wang et al., } \\
\text { 2020) }\end{array}$ \\
\hline Rhein & KM mouse & i.g. & 300 mg/kg (rhubarb extract) & $\begin{array}{l}\text { Liver, stomach, intestines, plasma, spleen, kidney, lung, } \\
\text { heart, fat, muscle, brain }\end{array}$ & $\begin{array}{l}\text { (Wang et al., } \\
\text { 2020) }\end{array}$ \\
\hline Rhein & SD rats & i.g. & $\begin{array}{l}2.0 \mathrm{~g} / \mathrm{kg} \text { of rheum palmatum } \mathrm{L} \text {. } \\
\text { decoction }\end{array}$ & Kidney, liver, lung & $\begin{array}{l}\text { (Shia et al., } \\
\text { 2011b) }\end{array}$ \\
\hline Physcion & $\mathrm{KM}$ mouse & i.g. & 300 mg/kg (rhubarb extract) & $\begin{array}{l}\text { Intestines, stomach, liver, lung, spleen, heart, plasma, } \\
\text { muscle, fat, brain, kidney }\end{array}$ & $\begin{array}{l}\text { (Wang et al., } \\
\text { 2020) }\end{array}$ \\
\hline
\end{tabular}

Regarding gastrointestinal diseases, aloe-emodin, rhein, rhein-8-O$\beta$-D-glycoside and sennoside A are distributed at higher levels in the liver and colon in the constipation model mice than in the normal group when they are treated with a Chinese formula, DahuangGancao decoction. The greater distribution in the colon may benefit the treatment of constipation (Chen et al., 2019). For acute pancreatitis, rhein in Da-Cheng-Qi decoction is distributed more in the pancreas than in normal rats, indicating a promising effect of Da-Cheng-Qi decoction on acute pancreatitis (Zhao et al., 2015). To investigate the potential change in the distribution of rhubarb anthraquinones, the total extract of Rhei Radix et Rhizoma was orally administered to normal and $\mathrm{CCl}_{4}$-induced liver injury rats. Data have shown that the distribution of aloe-emodin, emodin and rhein in the rat spleen, liver and kidney is decreased under liver injury (Fang et al., 2011), which deserves further study. The distribution of anthraquinones is listed in Table 3.

\section{METABOLISM}

Biotransformation is an important process for anthraquinones to be changed into inactive or more active metabolites and cleared from the body. The transformation occurs mainly in the liver. However, since most Chinese medicines are orally administered, biotransformation of anthraquinones has already begun in the early phase of absorption in the gut based on the actions of enzymes in the intestinal flora, including Bifidobacterium sp. (Wang et al., 2010), Peptostreptococcus, Clostridium spp., and Eubacteria (Rong et al., 2016). (Table 4 and Figure 1I).

\section{Hydrolysis}

Anthraquinone glycosides can be hydrolyzed by both intestinal bacteria and liver enzymes. Song et al. incubated processed rhubarb aqueous extracts with rat intestinal bacteria and found that 12 anthraquinone glycosides were hydrolyzed into anthraquinone aglycones, aloe-emodin, chrysophanol, emodin, and physcion respectively (Song et al., 2012) (Table 4). For anthraquinone glycoside-containing formulae, Liu and colleagues incubated XiaoCheng-Qi decoction (XCQD) with human intestinal bacteria in vitro and found that sennoside $\mathrm{A}$ and seven other anthraquinone glycosides were hydrolyzed (Liu X. Y. et al., 2018). It is worth noting that anthraquinone glycosides, such as aloe-emodin-8-O$\beta$-D-glucopyranoside, emodin-8-O- $\beta$-D-glucopyranoside, and rhein-8-O- $\beta$-D glucopyranoside can also be transformed into their aglycones by the enzymes in the liver (Xu et al., 2018).

\section{Glucuronidation}

Glucuronidation in the intestines and liver is one of the main phase II metabolic reactions of anthraquinones. UGTs play a pivotal role in the glucuronidation of anthraquinones (Wu et al., 2014; Meng and Ding, 2019). When oral administered with Zhi-Zi-Da-Huang decoction (ZZDHD), which consists of Gardenia jasminoides Ellis (Zhizi), Rheum palmatum L. (Dahuang), Citrus aurantium L. (Zhishi) and Sojae Semen Praeparatum (Dandouchi), emodin and rhein can be transformed to rhein-8-O-glucuronide, rhein-1-O-glucuronide, emodin-1-O-glucuronide, and emodin-3-O-glucuronide (Zhu et al,, 2015). Aloe-emodin is transformed to glucuronidation forms by $\beta$-glucuronidase and sulfatase/ $\beta$-glucuronidase following intravenous and oral administration in rats (Yu et al., 2016).

Da-Huang-Xiao-Shi decoction (DHXSD) is another formula for treating jaundice. It is composed of four crude drugs: Rheum officinale Baill (Dahuang), Gardenia jasminoides Ellis (Zhizi), Phellodendron amurense Rupr. (Huangbo), and Natrii Sulfas. When DHXSD was orally administered to rats, six anthraquinone glucuronidation, aloeemodin-O-glucuronide, chrysophanol-O-glucoside-O-glucuronide, rhein-O-glucuronide, 
TABLE 4 | Metabolic pathways and metabolites of anthraquinones.

\begin{tabular}{|c|c|c|c|c|c|c|}
\hline Compound & $\begin{array}{l}\text { Animal } \\
\text { species }\end{array}$ & Dose & $\begin{array}{l}\text { Administration } \\
\text { routines }\end{array}$ & Metabolic pathway & Metabolites & References \\
\hline Aloe-emodin & $\mathrm{SD}$ rats & $\begin{array}{l}10 \mathrm{~mL} / \mathrm{kg} \text { rhubarb } \\
\text { decoction }\end{array}$ & i.g. & $\begin{array}{l}\text { Glucuronidation, } \\
\text { hydroxylation, } \\
\text { hydrogenation, oxidation }\end{array}$ & $\begin{array}{l}\text { Aloe-emodin-8-O-glucoside-1-O- } \\
\text { glucuronide or aloe-emodin-1-O- } \\
\text { glucoside-8-O-glucuronide, 2- } \\
\text { hydroxyaloe-emodin- } \omega \text {-O-glucuronide }\end{array}$ & $\begin{array}{l}\text { (Song et al., } \\
\text { 2010) }\end{array}$ \\
\hline Aloe-emodin & SD rats & NA & Liver microsomes & $\begin{array}{l}\text { Monohydroxylation, } \\
\text { hydrogenation, } \\
\text { methylation, oxidation in } \\
\text { side chain }\end{array}$ & $\begin{array}{l}\text { Aloe-emodin, rhein, 1,8-dihydroxy-3- } \\
\text { hydroxymethyl-10-oxanthranol, 1,2,8- } \\
\text { trihydroxy-3- } \\
\text { hydroxymethylanthraquinon, 1,4,8- } \\
\text { trihydroxy-3- } \\
\text { hydroxymethylanthraquinon, } \\
\text { 1,8,9,10-tetrahydroxy-3-(methoxyl) } \\
\text { methyl-9,10-dihydroanthracene, 1,8- } \\
\text { dihydroxy-3-(methoxyl) } \\
\text { methylanthraquinone, 1,8-dihydroxy- } \\
\text { 3-hydroxymethyl-4- } \\
\text { methylanthraquinone, 1,8-dihydroxy- } \\
\text { 3-hydroxymethyl-2- } \\
\text { methylanthraquinone }\end{array}$ & $\begin{array}{l}\text { (Song et al., } \\
\text { 2009a) }\end{array}$ \\
\hline Aloe-emodin & $\mathrm{SD}$ rats & 0.035 mg/mL & Liver microsomes & $\begin{array}{l}\text { Hydroxylation, reduction, } \\
\text { oxidation }\end{array}$ & $\begin{array}{l}\text { Dihydroxy-aloe-emodin, hydroxy- } \\
\text { aloe-emodin, hydroxy-rhein, hydroxyl- } \\
\text { 1, 8-dihydroxy-3-hydroxymethyl-9- } \\
\text { oxanthranol/hydroxyl-1, 8-dihydroxy- } \\
\text { 3-hydroxymethyl-10-oxanthranol, } \\
\text { aloe-emodin, rhein isomer }\end{array}$ & (Xu et al., 2018) \\
\hline Aloe-emodin & $\mathrm{SD}$ rats & NA & Intestinal bacteria & $\begin{array}{l}\text { Hydrolysis, hydroxylation, } \\
\text { acetylation, demethylation }\end{array}$ & $\begin{array}{l}\text { 3-acetoxy-1,8-dihydroxy-6- } \\
\text { hydroxymethyl-10-oxanthranol, 2- } \\
\text { formyl-1,8-dihydroxy-3- } \\
\text { hydroxymethyl-6- } \\
\text { methoxyanthraquinone }\end{array}$ & $\begin{array}{l}\text { (Song et al., } \\
\text { 2011) }\end{array}$ \\
\hline Aloe-emodin & In vitro & 0.0156 mg/mL & $\begin{array}{l}\text { Human intestinal } \\
\text { bacteria }\end{array}$ & Reduction, methylation & $\begin{array}{l}\text { O-methyl-aloe-emodin, 1,8- } \\
\text { dihydroxy-3-hydroxymethyl-9- } \\
\text { oxanthranol or 1,8-dihydroxy-3- } \\
\text { hydro-xymethyl-10-oxanthranol and } \\
\text { aloe-emodin isomer }\end{array}$ & $\begin{array}{l}\text { (Huang et al., } \\
\text { 2019) }\end{array}$ \\
\hline $\begin{array}{l}\text { Aloe-emodin 1/8-O- } \\
\text { glycoside }\end{array}$ & In vitro & $0.5 \mathrm{~mL}$ & Intestinal bacteria & $\begin{array}{l}\text { Hydrolysis, reduction, } \\
\text { substitution reaction }\end{array}$ & $\begin{array}{l}\text { aloe-emodin, and reduction and } \\
\text { acetoxyl derivatives }\end{array}$ & $\begin{array}{l}\text { (Song et al., } \\
\text { 2012) }\end{array}$ \\
\hline $\begin{array}{l}\text { Aloe-emodin-8-O- } \\
\beta \text {-D-glycoside }\end{array}$ & $\mathrm{SD}$ rats & $0.0240 \mathrm{mg} / \mathrm{mL}$ & Liver microsomes & $\begin{array}{l}\text { Hydrolysis, hydroxylation, } \\
\text { reduction, oxidation }\end{array}$ & $\begin{array}{l}\text { aloe-emodin-8- O- } \beta \text {-D } \\
\text {-glucopyranoside, aloe-emodin } \\
\text { isomer, hydroxy-aloe-emodin, aloe- } \\
\text { emodin, rhein }\end{array}$ & (Xu et al., 2018) \\
\hline $\begin{array}{l}\text { Aloe-emdion- } \\
\text { O-glucopyranoside }\end{array}$ & In vitro & $\begin{array}{l}1 \mathrm{ml} \text { Xiao-Cheng-Qi } \\
\text { Decoction solution } \\
\text { (1 g/ml raw formula } \\
\text { herbs), including rhei } \\
\text { Radix et Rhizoma } \\
\text { (wine processed), } \\
\text { Aurantii Immaturus } \\
\text { Fructus and } \\
\text { Magnoliae officinalis } \\
\text { Cortex }\end{array}$ & $\begin{array}{l}\text { Human intestinal } \\
\text { bacteria }\end{array}$ & Hydrolysis and oxidation & aloe-emdion, rhein and rheinanthrone & $\begin{array}{l}\text { (Liu et al., } \\
\text { 2018b) }\end{array}$ \\
\hline $\begin{array}{l}\text { 11-O-actyl-aloe- } \\
\text { emdion-O- } \beta \text {-glc-xyl }\end{array}$ & In vitro & $\begin{array}{l}1 \mathrm{ml} \text { Xiao-Cheng-Qi } \\
\text { Decoction solution }\end{array}$ & $\begin{array}{l}\text { Human intestinal } \\
\text { bacteria }\end{array}$ & Hydrolysis and oxidation & $\begin{array}{l}\text { aloe-emdion, rhein and then } \\
\text { rheinanthrone }\end{array}$ & (Liu et al., 2018a) \\
\hline
\end{tabular}

$(1 \mathrm{~g} / \mathrm{ml}$ raw formula

herbs), including rhei

Radix et Rhizoma

(wine processed),

Aurantii Immaturus

Fructus and

Magnoliae officinalis

Cortex 
TABLE 4 | (Continued) Metabolic pathways and metabolites of anthraquinones.

\begin{tabular}{|c|c|c|c|c|c|c|}
\hline Compound & $\begin{array}{l}\text { Animal } \\
\text { species }\end{array}$ & Dose & $\begin{array}{l}\text { Administration } \\
\text { routines }\end{array}$ & Metabolic pathway & Metabolites & References \\
\hline Chrysophanol & $\mathrm{SD}$ rats & $\begin{array}{l}10 \mathrm{~mL} / \mathrm{kg} \text { rhubarb } \\
\text { decoction }\end{array}$ & i.g. & Glucuronidation, sulfation & $\begin{array}{l}\text { Chrysophanol-1-O-glucoside-8-O- } \\
\text { glucuronide, chrysophanol-8-O- } \\
\text { glucoside-1-O-glucuronide, } \\
\text { chrysophanol-1,8-biglucuronides, } \\
\text { chrysophanol-1-O-glucuronide, } \\
\text { chrysophanol-8-O-glucuronide }\end{array}$ & $\begin{array}{l}\text { (Song et al., } \\
\text { 2010) }\end{array}$ \\
\hline Chrysophanol & SD rats & $0.0755 \mathrm{mg} / \mathrm{mL}$ & Liver microsomes & $\begin{array}{l}\text { Hydroxylation, acetylation, } \\
\text { demethylation, } \\
\text { hydroxylation, reduction, } \\
\text { oxidation }\end{array}$ & $\begin{array}{l}\text { Chrysophanol, dihydroxy- } \\
\text { chrysophanol, dihydroxyl-1,8- } \\
\text { dihydroxy-3-methyl-9-oxanthranol/ } \\
\text { dihydroxyl-1,8-dihydroxy-3-methyl- } \\
\text { 10-oxanthranol, hydroxy- } \\
\text { chrysophanol, rhein }\end{array}$ & (Xu et al., 2018) \\
\hline Chrysophanol & $\mathrm{SD}$ rats & NA & Liver microsomes & $\begin{array}{l}\text { Monohydroxylation, } \\
\text { dihydroxylation }\end{array}$ & $\begin{array}{l}\text { Chrysophanol, 1,4,8-trihydroxy-3- } \\
\text { hydroxymethylanthraquinone, 2- } \\
\text { hydroxychrysophanol, 4- } \\
\text { hydroxychrysophanol }\end{array}$ & $\begin{array}{l}\text { (Song et al., } \\
\text { 2009a) }\end{array}$ \\
\hline Chrysophanol & SD rats & NA & Intestinal bacteria & $\begin{array}{l}\text { Hydrolysis, hydroxylation, } \\
\text { acetylation, demethylation }\end{array}$ & $\begin{array}{l}\text { 3-acetoxy-1,8-dihydroxy-6-methyl- } \\
\text { 10-oxanthanol, 1,8-dihydroxy-2- } \\
\text { (acetoxy) methyl-6- } \\
\text { methylanthraquinone, 1,8-dihydroxy- } \\
\text { 2-(1-hydroxyethoxy) methyl-6- } \\
\text { methylanthraquinone }\end{array}$ & $\begin{array}{l}\text { (Song et al., } \\
\text { 2011) }\end{array}$ \\
\hline Chrysophanol & In vitro & 0.0755 mg/mL & $\begin{array}{l}\text { Human intestinal } \\
\text { bacteria }\end{array}$ & $\begin{array}{l}\text { Reduction, hydrolysis, } \\
\text { acetylation, oxidation, } \\
\text { demethylation, methylation, } \\
\text { hydroxylation, } \\
\text { dehydroxylation }\end{array}$ & $\begin{array}{l}\text { Chrysophanol isomer, O-methyl- } \\
\text { hydroxy-chrysophanol, aloe-emodin, } \\
\text { O-methyl-chrysophanol, 1,8- } \\
\text { dihydroxy-3-methyl-9-oxanthranol or } \\
\text { 1,8-dihydroxy-3-methyl-10- } \\
\text { oxanthranol, emodin, acetyl-1,8-di- } \\
\text { hydroxy-anthraquinone, danthron, } \\
\text { rhein }\end{array}$ & $\begin{array}{l}\text { (Huang et al., } \\
\text { 2019; Tian., } \\
\text { et al., 2012) }\end{array}$ \\
\hline $\begin{array}{l}\text { Chrysophanol-1/8- } \\
\text { O-glucoside }\end{array}$ & In vitro & $0.5 \mathrm{~mL}$ & Intestinal bacteria & $\begin{array}{l}\text { Hydrolysis, reduction, } \\
\text { substitution reaction }\end{array}$ & $\begin{array}{l}\text { Chrysophanol and then reduction and } \\
\text { acetoxyl derivatives }\end{array}$ & $\begin{array}{l}\text { (Song et al., } \\
\text { 2012) }\end{array}$ \\
\hline $\begin{array}{l}\text { Chrysophanol-O- } \\
\text { glucopyranoside }\end{array}$ & In vitro & $\begin{array}{l}1 \mathrm{ml} \text { Xiao-Cheng-Qi } \\
\text { Decoction solution } \\
\text { (1 } \mathrm{g} / \mathrm{ml} \text { raw formula } \\
\text { herbs), including rhei } \\
\text { Radix et Rhizoma } \\
\text { (wine processed), } \\
\text { Aurantii Immaturus } \\
\text { Fructus and } \\
\text { Magnoliae officinalis } \\
\text { Cortex }\end{array}$ & $\begin{array}{l}\text { Human intestinal } \\
\text { bacteria }\end{array}$ & Hydrolysis and oxidation & $\begin{array}{l}\text { Chrysophanol, rhein and then } \\
\text { rheinanthrone }\end{array}$ & (Liu et al., 2018a) \\
\hline Emodin & $\begin{array}{l}\text { Wistar } \\
\text { rats }\end{array}$ & $50 \mathrm{mg} / \mathrm{kg}$ & i.g. & $\begin{array}{l}\text { Methylation, hydroxylation, } \\
\text { oxidation }\end{array}$ & $\begin{array}{l}\text { physcion, chrysophanol, aloe emodin, } \\
\text { danthron, rhein }\end{array}$ & $\begin{array}{l}\text { (Tian et al., } \\
\text { 2012) }\end{array}$ \\
\hline Emodin & SD rats & $\begin{array}{l}8 \mathrm{~g} / \mathrm{kg} \text { Zhi-Zi-Da- } \\
\text { Huang decoction }\end{array}$ & i.g. & Glucuronidation, sulfation & $\begin{array}{l}\text { Emodin-1-O-glucuronide, emodin-1- } \\
\text { O-sulfate, emodin-3-O-glucuronide, } \\
\text { emodin-3-O-sulfate }\end{array}$ & (Zhu et al., 2015) \\
\hline Emodin & SD rats & $2.26 \mathrm{mg} / \mathrm{kg}$ & i.g. & $\begin{array}{l}\text { Oxidation, acidification, } \\
\text { methylation, } \\
\text { glucuronidation, sulfation }\end{array}$ & $\begin{array}{l}\text { Emodin methylate, } \omega \text {-hydroxy- } \\
\text { emodin, 6-carboxyl emodin, physcion, } \\
\text { emodin, sulfonyl emodin, emodin-di- } \\
\text { glucuronide, emodin-glucuronide, } \\
\text { emodin-glucuronide oxidate, emodin- } \\
\text { sulfate oxidate }\end{array}$ & $\begin{array}{l}\text { (Zhang et al., } \\
\text { 2018b) }\end{array}$ \\
\hline
\end{tabular}


TABLE 4 | (Continued) Metabolic pathways and metabolites of anthraquinones.

\begin{tabular}{|c|c|c|c|c|c|c|}
\hline Compound & $\begin{array}{l}\text { Animal } \\
\text { species }\end{array}$ & Dose & $\begin{array}{l}\text { Administration } \\
\text { routines }\end{array}$ & Metabolic pathway & Metabolites & References \\
\hline Emodin & SD rats & $\begin{array}{l}10 \mathrm{~mL} / \mathrm{kg} \text { rhubarb } \\
\text { decoction }\end{array}$ & i.g. & $\begin{array}{l}\text { Glucuronidation, sulfation, } \\
\text { hydroxylation, } \\
\text { hydrogenation, oxidation }\end{array}$ & $\begin{array}{l}\text { emodin-O-diglucuronides, emodin-O- } \\
\text { glucoside-O-glucuronide, 1,8- } \\
\text { Dihydroxy-3-carboxy-6- } \\
\text { methylanthraquinone-1or 8-O- } \\
\text { glucoside, emodin-1 or 8-O- } \\
\text { glucuronide-3-O-sulfate or emodin-1 } \\
\text { or 8-O-sulfate-3-O-glucuronide, } \\
\text { 1,3,8-trihydroxy-6-methyl-10- } \\
\text { oxanthranol glucuronide, emodin-O- } \\
\text { diglucuronides, 1,3,8-trihydroxy-6- } \\
\text { (glucuronidyl)methylanthrquinone, } \\
\text { emodin acid-O-glucuronide, emodin- } \\
\text { 2-C-glucuronide, emodin-3-O- } \\
\text { glucuronide }\end{array}$ & $\begin{array}{l}\text { (Song et al., } \\
\text { 2010) }\end{array}$ \\
\hline Emodin & SD rats & $\begin{array}{l}\text { Raw root of } P \text {. } \\
\text { multiflorum Thunb } \\
\text { extract ( } 10 \mathrm{~mL} / \mathrm{kg}^{\prime} \\
2 \mathrm{~g} / \mathrm{mL})\end{array}$ & i.g. & $\begin{array}{l}\text { Glucuronidation, sulfation, } \\
\text { oxidation }\end{array}$ & $\begin{array}{l}\text { Emodin glucuronide sulfate, emodin 1, } \\
\text { 8-O-diglucuronide, emodin 1, 3-O- } \\
\text { diglucuronide, emodin 3, 8-O- } \\
\text { diglucuronide, 4-hydroxyemodin, 5- } \\
\text { hydroxyemodin, emodin acid-3-O- } \\
\text { glucuronide, emodin acid-3-O-sulfate, } \\
\text { physcion-glucuronides }\end{array}$ & $\begin{array}{l}\text { (Huang et al., } \\
\text { 2018) }\end{array}$ \\
\hline Emodin & SD rats & 0.0156 mg/mL & Liver microsomes & $\begin{array}{l}\text { Transhydroxylation, } \\
\text { hydroxylation, reduction, } \\
\text { dehydroxylation, oxidation }\end{array}$ & $\begin{array}{l}\text { Hydroxy-emodin, 1,3,8-trihydroxy-6- } \\
\text { methyl-9-oxanthranol/1, 3,8- } \\
\text { trihydroxy-6-methyl -10-oxanthranol, } \\
\text { dihydroxy-emodin, hydroxy-emodin, } \\
\text { aloe-emodin isomer, hydroxy-rhein, } \\
\text { hydroxyl-aloe-emodin, aloe-emodin, } \\
\text { emodin }\end{array}$ & (Xu et al., 2018) \\
\hline Emodin & SD rats & NA & Liver microsomes & Hydroxylation & $\begin{array}{l}\text { w-hydroxyemodin, 2-hydroxyemodin, } \\
\text { 4-hydroxyemodin, emodin acid, 3- } \\
\text { carbomethoxy-6-methoxy-1,8- } \\
\text { dihydroxyanthraquinone, physcion }\end{array}$ & $\begin{array}{l}\text { (Song et al., } \\
\text { 2008) }\end{array}$ \\
\hline Emodin & $\mathrm{SD}$ rats & NA & $\begin{array}{l}\text { Liver microsomes/ } \\
\text { intestinal bacteria }\end{array}$ & $\begin{array}{l}\text { Monohydroxylation, } \\
\text { methylation, oxidation in } \\
\text { side chain }\end{array}$ & $\begin{array}{l}\text { Emodin, physcion, 1, 3, 8-trihydroxy- } \\
\text { 6-(acetoxy) methyl-10-oxanthranol, } \\
\text { w-hydroxyemodin, 2-hydroxyemodin, } \\
\text { 4-hydroxyemodin, emodin acid, 3- } \\
\text { carbomethoxy-6-methoxy-1,8- } \\
\text { dihydroxyanthraquinone, 1,8- } \\
\text { dihydroxy-3-hydroxymethyl-10- } \\
\text { oxanthranol }\end{array}$ & $\begin{array}{l}\text { (Song et al., } \\
\text { 2008; Song } \\
\text { et al., 2009a; } \\
\text { Song et al., } \\
\text { 2011) }\end{array}$ \\
\hline Emodin & In vitro & $0.1950 \mathrm{mg} / \mathrm{mL}$ & $\begin{array}{l}\text { Human intestinal } \\
\text { bacteria }\end{array}$ & $\begin{array}{l}\text { Acetylation, hydroxylation, } \\
\text { methylation, trans } \\
\text { hydroxylation, reduction }\end{array}$ & $\begin{array}{l}\text { Aloe-emodin, isomer of emodin, 8-O- } \\
\text { methyl-emodin, 1-O-methyl- } \\
\text { emodin,3-O-methyl-emodin, 2- } \\
\text { hydroxy-emodin, 4-hydroxy-emodin, } \\
\text { w-hydroxy-emodin, acetyl-1,3,8- } \\
\text { trihydroxy-6-methyl-9-oxan-thranol or } \\
\text { acetyl-1,3,8-trihydroxy-6-methyl-10- } \\
\text { oxanthranol, acetyl-hydroxy-emodin }\end{array}$ & $\begin{array}{l}\text { (Huang et al., } \\
\text { 2019) }\end{array}$ \\
\hline $\begin{array}{l}\text { Emodin-1/8- } \\
\text { O-glucoside }\end{array}$ & In vitro & $0.5 \mathrm{~mL}$ & Intestinal bacteria & $\begin{array}{l}\text { Hydrolysis, reduction, } \\
\text { substitution reaction }\end{array}$ & $\begin{array}{l}\text { Emodin and then reduction and } \\
\text { acetoxyl derivatives }\end{array}$ & $\begin{array}{l}\text { (Song et al., } \\
\text { 2012) }\end{array}$ \\
\hline $\begin{array}{l}\text { Emodin-8-O- } \beta-D- \\
\text { glucoside }\end{array}$ & $\mathrm{SD}$ rats & $0.01 \mathrm{mg} / \mathrm{mL}$ & Liver microsomes & $\begin{array}{l}\text { Transhydroxylation, } \\
\text { hydrolysis, oxidation, } \\
\text { hydroxylation }\end{array}$ & $\begin{array}{l}\text { Dihydroxyl-1, 3, 8-trihydroxy-6- } \\
\text { methyl-9-oxanthranol/dihydroxyl-1, 3, } \\
\text { 8-trihydroxy-6-methyl-10- } \\
\text { oxanthranol, hydroxy-emodin-O- } \\
\text { glucopyranoside, hydroxy-emodin-O- } \\
\text { glucopyranoside, emodin-8-O- } \\
\beta \text {-glucopyranoside, emodin }\end{array}$ & (Xu et al., 2018) \\
\hline
\end{tabular}

(Continued on following page) 
TABLE 4 | (Continued) Metabolic pathways and metabolites of anthraquinones.

\begin{tabular}{|c|c|c|c|c|c|c|}
\hline Compound & $\begin{array}{l}\text { Animal } \\
\text { species }\end{array}$ & Dose & $\begin{array}{l}\text { Administration } \\
\text { routines }\end{array}$ & Metabolic pathway & Metabolites & References \\
\hline $\begin{array}{l}\text { Emodin-O- } \\
\text { glucopyranoside }\end{array}$ & In vitro & $\begin{array}{l}1 \mathrm{ml} \text { Xiao-Cheng-Qi } \\
\text { Decoction solution } \\
\text { (1 } \mathrm{g} / \mathrm{ml} \text { raw formula } \\
\text { herbs), including rhei } \\
\text { Radix et Rhizoma } \\
\text { (wine processed), } \\
\text { Aurantii Immaturus } \\
\text { Fructus and } \\
\text { Magnoliae officinalis } \\
\text { Cortex }\end{array}$ & $\begin{array}{l}\text { Human intestinal } \\
\text { bacteria }\end{array}$ & Hydrolysis and oxidation & Emodin, rhein and then rheinanthrone & (Liu et al., 2018a) \\
\hline Physcion & SD rats & NA & i.g. & Glucuronidation, sulfation & $\begin{array}{l}\text { Physcion oxidate, physcion-sulfate, } \\
\text { physcion-glucuronide }\end{array}$ & $\begin{array}{l}\text { (Zhang et al., } \\
\text { 2018a) }\end{array}$ \\
\hline Physcion & $\mathrm{SD}$ rats & $\begin{array}{l}10 \mathrm{~mL} / \mathrm{kg} \text { rhubarb } \\
\text { decoction }\end{array}$ & i.g. & Glucuronidation, sulfation & $\begin{array}{l}\text { Physcion-1-O-glucoside-8-O- } \\
\text { glucuronide or physcion-8-O- } \\
\text { glucoside-1-O-glucuronide, physcion- } \\
\text { 1, 8-O-diglucuronides }\end{array}$ & $\begin{array}{l}\text { (Song et al., } \\
2010 \text { ) }\end{array}$ \\
\hline Physcion & SD rats & NA & Liver microsomes & $\begin{array}{l}\text { Monohydroxylation, } \\
\text { oxidation in side chain, } \\
\text { demethylation }\end{array}$ & $\begin{array}{l}\text { Emodin, 1,8-dihydroxy-3- } \\
\text { methoxyanthraquinone, 1,8- } \\
\text { dihydroxy-3-hydroxymethyl-6- } \\
\text { methoxyanthraquinone, } \\
\text { hydroxyphyscion, emodin acid, } \\
\text { w-hydroxyemodin, 4-hydroxyemodin, } \\
\text { 3-carbomethoxy-6-methoxy-1,8- } \\
\text { dihydroxyanthraquinone }\end{array}$ & $\begin{array}{l}\text { (Song et al., } \\
\text { 2009a) }\end{array}$ \\
\hline Physcion & SD rats & $0.16 \mathrm{mg} / \mathrm{mL}$ & Liver microsomes & $\begin{array}{l}\text { Demethylation, } \\
\text { hydroxylation, reduction }\end{array}$ & $\begin{array}{l}\text { Dihydroxy-1,8-dihydroxy-3-methoxy- } \\
\text { 6-methyl-9-oxanthranol/1, 8- } \\
\text { dihydroxy-3-methoxy-6- methyl-10- } \\
\text { oxanthranol, emodinlsomer, hydroxy- } \\
\text { emodin, emodin, physcion }\end{array}$ & (Xu et al., 2018) \\
\hline Physcion & SD rats & NA & Intestinal bacteria & $\begin{array}{l}\text { Hydrolysis, hydroxylation, } \\
\text { acetylation, demethylation }\end{array}$ & $\begin{array}{l}\text { 2-Formyl-1,8-dihydroxy-3- } \\
\text { hydroxymethyl-6- } \\
\text { methoxyanthraquinone, 1,8- } \\
\text { dihydroxy-2-(acetoxy) methyl-3- } \\
\text { methoxyanthraquinone, 3-acetoxy } \\
\text {-1,8-dihydroxy-6-(acetyl) } \\
\text { methylanthraquinone }\end{array}$ & $\begin{array}{l}\text { (Song et al., } \\
\text { 2011) }\end{array}$ \\
\hline Physcion & In vitro & $0.1610 \mathrm{mg} / \mathrm{mL}$ & $\begin{array}{l}\text { Human intestinal } \\
\text { bacteria }\end{array}$ & $\begin{array}{l}\text { demethylation, } \\
\text { dehydroxylation, } \\
\text { transhydroxylation }\end{array}$ & $\begin{array}{l}\text { Chrysophanol isomer, physcion } \\
\text { isomer, aloe-emodin, emodin }\end{array}$ & $\begin{array}{l}\text { (Huang et al., } \\
\text { 2019) }\end{array}$ \\
\hline $\begin{array}{l}\text { Physcion-O- } \\
\text { glucoside }\end{array}$ & In vitro & $0.5 \mathrm{~mL}$ & Intestinal bacteria & $\begin{array}{l}\text { Hydrolysis, reduction, } \\
\text { substitution reaction }\end{array}$ & $\begin{array}{l}\text { physcion and then reduction and } \\
\text { acetoxyl derivatives }\end{array}$ & $\begin{array}{l}\text { (Song et al., } \\
\text { 2012) }\end{array}$ \\
\hline Rhein & SD rats & $\begin{array}{l}8 \mathrm{~g} / \mathrm{kg} \text { Zhi-Zi-Da- } \\
\text { Huang decoction }\end{array}$ & i.g. & glucuronidation, sulfation & $\begin{array}{l}\text { Rhein-1-O-sulfate, rhein-8-O-sulfate, } \\
\text { rhein-8-O-glucuronide, rhein-1-O- } \\
\text { glucuronide }\end{array}$ & (Zhu et al., 2015) \\
\hline Rhein & SD rats & $\begin{array}{l}10 \mathrm{~mL} / \mathrm{kg} \text { rhubarb } \\
\text { decoction }\end{array}$ & i.g. & glucuronidation, sulfation & rhein, rhein-1-O-glucoside & $\begin{array}{l}\text { (Song et al., } \\
\text { 2010) }\end{array}$ \\
\hline Rhein & SD rats & NA & Liver microsomes & Hydrogenation, methylation & $\begin{array}{l}\text { 1,8-dihydroxy-3-carboxy-9- } \\
\text { oxanthranol, 1,8-dihydroxy-3- } \\
\text { carboxy-10-oxanthranol, 2- } \\
\text { methylrhein }\end{array}$ & $\begin{array}{l}\text { (Song et al., } \\
\text { 2009a) }\end{array}$ \\
\hline Rhein & SD rats & 0.1950 mg/mL & Liver microsomes & Hydroxylation, reduction & $\begin{array}{l}\text { rhein, rhein isomer, dihydroxyl-1,8- } \\
\text { dihydroxy-3-carboxyl-9-oxanthranol/ } \\
\text { dihydroxyl-1,8-dihydroxy-3-carboxyl- } \\
\text { 10-oxanthranol }\end{array}$ & (Xu et al., 2018) \\
\hline Rhein & SD rats & NA & Intestinal bacteria & $\begin{array}{l}\text { Hydrolysis, hydroxylation, } \\
\text { acetylation, demethylation }\end{array}$ & $\begin{array}{l}\text { 2-acetoxy -6-carboxy -1,8- } \\
\text { dihydroxyanthraquinone, 3- } \\
\text { acetoxy-1,8-dihydroxy-6- } \\
\text { hydroxymethyl-10-oxanthranol }\end{array}$ & $\begin{array}{l}\text { (Song et al., } \\
\text { 2011) }\end{array}$ \\
\hline Rhein & In vitro & 0.0350 mg/mL & $\begin{array}{l}\text { Human intestinal } \\
\text { bacteria }\end{array}$ & $\begin{array}{l}\text { methylation, hydroxylation, } \\
\text { reduction }\end{array}$ & $\begin{array}{l}\text { rhein, O-methyl-rhein, 1,8-dihydroxy- } \\
\text { 3-carboxyl-9-oxanthranol, 1,8- } \\
\text { dihydroxy-3-carboxyl-10-oxanthranol, } \\
\text { hydroxy-rhein, chrysophanol isomer }\end{array}$ & $\begin{array}{l}\text { (Huang et al., } \\
\text { 2019) }\end{array}$ \\
\hline
\end{tabular}

(Continued on following page) 
TABLE 4 | (Continued) Metabolic pathways and metabolites of anthraquinones.

\begin{tabular}{|c|c|c|c|c|c|c|}
\hline Compound & $\begin{array}{l}\text { Animal } \\
\text { species }\end{array}$ & Dose & $\begin{array}{l}\text { Administration } \\
\text { routines }\end{array}$ & Metabolic pathway & Metabolites & References \\
\hline Rhein & In vitro & $\begin{array}{l}1 \mathrm{ml} \text { Xiao-Cheng-Qi } \\
\text { Decoction solution } \\
\text { (1g/ml raw formula } \\
\text { herbs), including rhei } \\
\text { Radix et Rhizoma } \\
\text { (wine processed), } \\
\text { Aurantii Immaturus } \\
\text { Fructus and } \\
\text { Magnoliae officinalis } \\
\text { Cortex }\end{array}$ & $\begin{array}{l}\text { Human intestinal } \\
\text { bacteria }\end{array}$ & Hydrolysis & Rheinanthrone & (Liu et al., 2018a) \\
\hline $\begin{array}{l}\text { Rhein-8-O- } \\
\text { glucoside }\end{array}$ & SD rats & $0.025 \mathrm{mg} / \mathrm{mL}$ & Liver microsomes & $\begin{array}{l}\text { Hydrolysis, hydroxylation, } \\
\text { reduction }\end{array}$ & $\begin{array}{l}\text { Rhein-8-O-glucopyranoside, } \\
\text { dihydroxy-3-carboxyl-9-oxanthranol- } \\
\text { O-glucopyranoside/1, 8-dihydroxy-3- } \\
\text { carboxyl-10-oxanthranol-O- } \\
\text { glucopyranoside, rhein, emodin } \\
\text { isomer }\end{array}$ & (Xu et al., 2018) \\
\hline Sennoside A & Human & $0.0250 \mathrm{mg} / \mathrm{mL}$ & Intestinal bacteria & $\begin{array}{l}\text { Hydrolysis, methylation, } \\
\text { hydroxylation, } \\
\text { dehydroxylation, reduction }\end{array}$ & $\begin{array}{l}\text { sennidine A-8-O-monoglucoside, } \\
\text { rheinanthrone, dehydroxy- } \\
\text { rheinanthrone, O-methyl-hydroxy- } \\
\text { rheinanthrone, rhein }\end{array}$ & $\begin{array}{l}\text { (Huang et al., } \\
\text { 2019) }\end{array}$ \\
\hline sennoside A & In vitro & $\begin{array}{l}1 \mathrm{ml} \text { Xiao-Cheng-Qi } \\
\text { Decoction solution } \\
\text { (1g/ml raw formula } \\
\text { herbs), including rhei } \\
\text { Radix et Rhizoma } \\
\text { (wine processed), } \\
\text { Aurantii Immaturus } \\
\text { Fructus and } \\
\text { Magnoliae officinalis } \\
\text { Cortex }\end{array}$ & $\begin{array}{l}\text { Human intestinal } \\
\text { bacteria }\end{array}$ & Hydrolysis & Rheinanthrone & (Liu et al., 2018a) \\
\hline Sennoside B & Human & $0.0393 \mathrm{mg} / \mathrm{mL}$ & 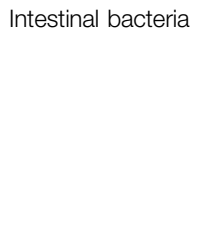 & $\begin{array}{l}\text { Hydrolysis, methylation, } \\
\text { hydroxylation, } \\
\text { dehydroxylation, reduction }\end{array}$ & $\begin{array}{l}\text { Sennoside A, dehydroxy- } \\
\text { rheinanthrone, O-methyl- } \\
\text { rheinanthrone, sennidine B-8-O- } \\
\text { monoglucoside, sennidine A-8-O- } \\
\text { monoglucoside, aloe-emodin, } \\
\text { O-methyl-hydroxy-rheinanthrone, } \\
\text { O-methyl-rheinanthrone, rhein }\end{array}$ & $\begin{array}{l}\text { (Huang et al., } \\
\text { 2019) }\end{array}$ \\
\hline Sennoside C & Human & 0.0398 mg/mL & Intestinal bacteria & $\begin{array}{l}\text { Hydrolysis, oxidation, } \\
\text { methylation, } \\
\text { dehydroxylation, reduction }\end{array}$ & $\begin{array}{l}\text { sennoside C, sennidine C-8- } \\
\text { monoglucoside, sennidine C-8'- } \\
\text { monoglucoside, rheinanthrone-8-O- } \\
\text { monoglucoside, dehydroxy- } \\
\text { rheinanthrone, rhein, aloe-emodin, } \\
\text { O-methyl- rheinanthrone }\end{array}$ & $\begin{array}{l}\text { (Huang et al., } \\
\text { 2019) }\end{array}$ \\
\hline Sennoside D & Human & $0.0263 \mathrm{mg} / \mathrm{mL}$ & Intestinal bacteria & $\begin{array}{l}\text { Hydrolysis, oxidation, } \\
\text { methylation, } \\
\text { dehydroxylation, reduction }\end{array}$ & $\begin{array}{l}\text { Chrysophanol isomer, sennidine D-8- } \\
\text { O-monoglucoside or sennidine D-8'- } \\
\text { O-monoglucoside, O-methyl- } \\
\text { rheinanthrone, aloe-emodin, rhein }\end{array}$ & $\begin{array}{l}\text { (Huang et al., } \\
\text { 2019) }\end{array}$ \\
\hline
\end{tabular}

NA: not available; i. g.: intragastrical administration; I.V.: intravenous injection.

physcion-O-glucoside-O-glucuronide, chrysophanol-Oglucuronide, and emodin-O-glucuronide were transformed to glucuronidation forms (Wang D. et al., 2017) (Table 4).

\section{Sulfonation}

Sulfonation in the intestines and liver is the other main phase II metabolic reaction of anthraquinones by sulfotransferase (SULT) (Song, et al., 2010). Like glucuronidation, the sulfonation is another detoxification process. Additionally, sulfonated anthraquinones can be used as a remedy strategy for free radical-related diseases such as AAPH $\left(2,2^{\prime}\right.$-azobis (2- amidinopropane hydrochloride))-induced hemolysis (Shia et al., 2009; Shia et al., 2010).

Aloe-emodin, chrysophanol, emodin, physcion, and rhein are metabolized to sulfonation forms (Song, et al., 2010; Zhu et al., 2015; Zhang, et al., 2018a; Huang et al., 2018). This can lead to a decline in the oral bioavailability of anthraquinones (Teng et al., 2007; Shia et al., 2009) (Table 4).

\section{Methylation/Demethylation}

Methylation is another metabolic reaction for anthraquinones in both the intestines and the liver (Song Z. et al., 2009). Aloe- 
emodin (Song R. et al., 2009), chrysophanol (Huang et al., 2019), emodin (Tian et al., 2012), rhein (Song R. et al., 2009; Huang et al., 2019) and rheinanthrone (Huang et al., 2019) are methylated to O-methyl-aloe-emodin, O-methyl-chrysophanol, 8-O-methylemodin, O-methyl-rhein, and O-methyl-rheinanthrone, respectively. O-methyltransferase may be involved in the methylation process (Koyama et al., 2009; Huang et al., 2019). Conversely, demethylation is an opposite reaction in anthraquinone metabolic processes. The demethylation of chrysophanol is transformed to dihydroxy-chrysophanol, while physcion is transformed to emodin/isomer (Xu et al., 2018). Of note, the rapid demethylation of physcion to emodin may be the reason why the bioavailability of physcion is low (Song $\mathrm{R}$. et al., 2009).

\section{Hydroxylation/Dehydroxylation}

The hydroxylation of emodin is hydroxy-emodin and dihydroxyemodin. Chrysophanol can also be transformed to hydroxylation forms as hydroxy-chrysophanol and dihydroxy-chrysophanol (Xu et al., 2018). Hydroxylation is also the synthesis pathway to form anthraquinone glycosides. Additionally, aleo-emodin is transformed to aloe-emodin-8-O-glucoside-1-O-glucuronide or aloe-emodin-1-O-glucoside-8-O-glucuronide, 2-hydroxyaloeemodin- $\omega$-O-glucuronide through hydroxylation, glucuronidation, hydrogenation, and oxidation (Song et al., 2010). Cytochromosome P450s, including CYP1A2, CYP2C19, CYP2B6, and CYP3A4, play major roles in the hydroxylation of anthraquinones (He et al., 2015; Qin et al., 2018). In contrast, emodin (Xu et al., 2018) and rheinanthrone (Huang et al., 2019) are dehydroxylated to chrysophanol isomers, and dehydroxyrheinanthrone, respectively. Other hydroxylation and dehydroxylation are listed in Table 4.

\section{Oxidation/Reduction (Hydrogenation)}

For oxidation, chrysophanol (Xu et al., 2018), emodin (Zhang J. et al., 2018), physcion (Song R. et al., 2009), rheinanthrone (Huang et al., 2019) and aloe-emodin anthrone (Huang et al., 2019) are oxidized to $\omega$-hydroxy-emodin, rhein and aloeemodin in the intestines and liver. Aloe-emodin (Song R. et al., 2009; Song et al., 2010; Xu et al., 2018) is oxidized to rhein. The oxidation reaction can decrease the bioavailability of anthraquinones. The order of bioavailability of some anthraquinones is: rhein $>$ emodin $>$ chrysophanol $>$ aloeemodin. This may result from that sennosides A and B, aloeemodin and chrysophanol all being oxidized to rhein (Shia et al., 2011a). CYP1A2, CYP2B6 and CYP3A4 are the major enzymes for oxidation (Sun et al., 2018).

For reduction, aloe-emodin, chrysophanol, emodin, physcion, rhein and rhein-8-O-glycopyranoside are hydrogenated (Xu et al., 2018; Yu et al., 2018; Huang et al., 2019). (Table 4).

\section{Acetylation}

Chrysophanol, emodin, physcion and rhein can be acetylated into acetyl-1,8-dihydroxy-anthraquinone, acetyl-1,3,8-trihydroxy-6methyl-9-oxanthranol and 1,8-dihydroxy-2-(acetoxy) methyl3-methoxyanthraquinone, respectively (Song et al., 2011; Xu et al., 2018; Huang et al., 2019).

\section{Esterification}

Rhein is Esterified to Rhein Methyl Ester by intestinal Flora (Fan et al., 2016).

\section{Affecting Factors Physiological Condition}

Sex The glucuronidation of emodin shares the same rate in human males and females, while the rates in females are faster than the rates in male rats, guinea pigs, and dogs. However, at an emodin concentration of $2.5 \mu \mathrm{M}$, male mice have a higher rate of glucuronidation than females (Liu et al., 2010). In addition, danthron and chrysophanol produced from emodin metabolism are only present in male rats (Tian et al., 2012). The bioavailability of rhein in female rats is higher than that in males. The mechanism may be the different activation of UGTs between the male and female (Zhang et al., 2015).

\section{Disorders}

The glucuronidation and hydrolysis of anthraquinones and their glycosides are reduced in rats with ulcerative colitis. The mechanism may be that colitis reduces the activities of $\beta$-glucosidases and $\beta$-glucuronidases in the intestinal flora ( $\mathrm{Wu}$ W. J. et al., 2017). In alcohol-induced liver injury, the metabolism of aloe-emodin, chrysophanol, physcion, aurantio-obtusin, chrysoobtusin, emodin, obtusin and rhein increase. This may result from that alcohol induces P450 (e.g., CTP2E1, CYP3A and CYP1A) (Shao and Feng, 2015; Li P. et al., 2017). Furthermore, the metabolism of rhein decreases under acute liver injury because of the lower expression and activity of CYP450, especially in males (Zhang et al., 2015).

\section{Drugs}

Drug-Drug Interactions Preparations with wine are very common for Chinese medicines. Thus the role of wine (ethanol) in Chinese medicines has attracted more research interest. Studies have shown that Rhei Radix et Rhizoma steamed with wine can accelerate the hydrolysis of anthraquinone glycosides in rats. This results in higher bioavailability of emodin, physcion and chrysophanol (Zhang et al., 2019). Additionally, wine reduces the $T_{1 / 2}$ of aloeemodin and emodin in Rhei Radix et Rhizoma (Wu Y. et al., 2017). This may be consistent with the traditional Chinese medicine theory of drug processing (known as Paozhi): wine promotes blood circulation. It is very common for ethanol to be used for drug processing of Chinese medicine to induce bioavailability, enhance efficacy and/or decrease adverse drug reactions.

For anthraquinone compounds, piperine increases the AUC and $C_{\max }$ of emodin by inhibiting UGTs (Di et al., 2015). Synergism can also occur between different anthraquinones. Sennoside $\mathrm{A}$ is an active anthraquinone glucoside in rhubarb (Rhei Radix et Rhizoma) for treating constipation. Rhein 8-O$\beta$-D-glucopyranoside, emodin, aloe-emodin and rhein can enhance the purgative action of sennoside A by accelerating its hydrolysis by inducing intestinal bacteria (Takayama et al., 2012).

Furthermore, the different classes of compounds in the same herb may influence the PKs of anthraquinones. 2,3,5,4Tetrahydroxy-stilbene-2-O- $\beta$-D-glycoside (TSG), a compound in Polygini Multiflori Radix (Heshouwu) (Li et al., 2016; Li H. 
et al., 2017) inhibits the mRNA expression of the UGT isoforms, UGT1A8, UGT1A10, and UGT12B7, leading to a decrease in glucuronidation of emodin (Ma et al., 2013; Yu et al., 2017). Inhibiting emodin glucuronidation will increase the bioavailability of emodin; however, it also leads to an accumulation of emodin to induce liver damage (Ma et al., 2015). Interestingly, TSG also accelerates metabolism to clear emodin by enhancing the activity of CYP1A2 (Xing et al., 2019), indicating that the interaction role of TSG in emodin pharmacological and toxicological actions is complex and needs to be further studied.

Rhei Radix et Rhizoma exerts purgative action for constipation. However, hepatotoxicity and abdominal pain limit its clinical application. When using Rhei Radix et Rhizoma combination with Glycyrrhizae Radix et Rhizoma (Gancao) (Da-HuangGancao Decoction in Chinese, Daiokanzoto in Japanese), hepatotoxicity and abdominal pain were reduced. The underlying mechanisms may be due to Glycyrrhizae Radix et Rhizoma inducing P450 to accelerate the transformation of emodin (Han et al., 2010). Furthermore, liquiritin and liquiritin apioside in Glycyrrhizae Radix et Rhizoma can induce intestinal bacteria to intensify the metabolism of sennoside A and enhance purgative action (Matsui et al., 2011). Increasing research on the intestinal flora may provide more insights into the novel role of intestinal bacteria in the PKs of anthraquinones.

Dahuang Fuzi decoction is the combination of Rhei Radix et Rhizoma, Aconiti Lateralis Radix Praeparata (Fuzi) and Asari Radix et Rhizoma (Xixin). Drug extrusion by intestinal P-gp can both reduce drug absorption and modulate the effects of inhibitors and inducers of CYP3A/CYP3A4-mediated metabolism. The study has shown that the compounds from Aconiti Lateralis Radix Praeparata or Asari Radix et Rhizoma may induce P-gp and CYP3A/CYP3A4, leading to a decrease in AUC and $C_{\max }$ for anthraquinones (Liu et al., 2015).

Xin et al. reported that San-Huang-Xie-Xin decoction (SHXXD), including Rhei Radix et Rhizoma, Scutellariae Radix and Coptidis Rhizoma (containing berberine), showed increases in the $C_{\max }$ and AUC of rhein compared with the single herb Rhei Radix et Rhizoma (Xin et al., 2009). The mechanisms may be due to the inhibited glucuronidation activity of UGTs for rhein by other ingredients in SHXXD (Hou et al., 2014).

The metabolic pathways and metabolites of anthraquinones are listed in Table 4.

\section{EXCRETION}

\section{Excretion Routes and Form}

Generally, anthraquinones are mainly excreted via the kidney (Chen et al., 2014), recta (Zhang M. et al., 2018), and/or gallbladder (Ma et al., 2005) via prototypes and/or metabolites. They are excreted with urine (Ma et al., 2005), feces (Zhang J. et al., 2018), and/or bile (Ma et al., 2005).

Anthraquinones excreted through bile may be reabsorbed and utilized in the intestines to form a hepatointestinal circulation, so they can be excreted for a long time (Yang B. et al., 2019). The amount of chrysophanol excreted through urine is significantly greater than that excreted through bile (Ma et al., 2005). The urinary excretion of emodin is 1.5-folds that of feces (Sun et al., 1986; Wu et al., 2008; Du et al., 2014). Regarding metabolite elimination of anthraquinones, e.g., rhein, the metabolite of emodin, exists in the plasma for a short time because of the rapid excretion (Tian et al., 2012).

Glucuronic acid and sulfuric acid conjugates of rhein are dominant in urine and fecal excreta. Only $20 \%$ of the prototype rhein is excreted in urine and feces (Wan et al., 2013).

\section{Affecting Factors Physiological Condition}

Species Physcion can be detectable in the urine of humans rather than in that of rats. However, there is an opposite result for rhein between humans and rats. In addition to differences in dosage and detection instruments, this species diversity may result from apparent distribution volume (Li et al., 2003).

Sex The excretion of danthron and rhein in male rats is faster than that in female (Tian et al., 2012). The excretion of emodin glucuronide is slower in male rats than that in female rats (Liu W. et al., 2011).

Food Feeding increases the half times of elimination $\left(T_{1 / 2}\right)$ of emodin and rhein, possibly because feeding stimulates an increase in bile secretion to form hepato-intestinal circulation. Additionally, feeding inhibits the activity and the saturation of the related metabolic enzymes and consequently increases the $T_{1 / 2}$ of emodin and rhein (Gong et al., 2011).

\section{Disorders}

The mean residence times (MRTs) of anthraquinones, e.g., aloeemodin, chrysophanol, emodin, physcion, and rhein are prolonged in microcirculation disorder (Dai et al., 2014; Yan and Dai, 2014; Zhu et al., 2017). For ischemic cerebrovascular disease, the elimination s of aloe-emodin, emodin, and rhein are significantly decreased in thrombotic cerebral ischemia compared with normal condition in rats (Feng et al., 2013). The $T_{1 / 2}$ values of chrysophanol and rhein are increased in acute pancreatitis, and the plasma clearance rates (CL) are decreased (Gong et al., 2009; Yang et al., 2012). Regarding liver disorders, the MRT of rhein is shortened and elimination is accelerated in acute liver injury rats (Zhang et al., 2015). However, in the other reports, the $\mathrm{T}_{1 / 2}$ values of aloe-emodin, chrysophanol, emodin and rhein increase (Li P. et al., 2017; Yang N. et al., 2019). The contradict results may result from the different animal models. For alcoholic liver injury, the $\mathrm{T}_{1 / 2}$ and MRT of emodin in rats are prolonged, and CL is decreased (Zhu et al., 2016). In addition, studies have reported that gastrointestinal disorders caused by alcoholic liver injury may affect the excretion of drugs (Burkard et al., 2005; Luo et al., 2014). The $T_{1 / 2}$ of chrysophanol and rhein increases in rats with ulcerative colitis (Wu W. J. et al., 2017). Under chronic renal failure conditions, the elimination of rhein is accelerated in rats due to urine alkalization and an increase in urine output (Wang et al., 2009).

The $\mathrm{T}_{1 / 2}$ values of chrysophanol and rhein in Rhei Radix et Rhizoma are increased in lipopolysacchoride (LPS)-induced inflammation. However, the underlying mechanisms are unkown (Li et al., 2013c). 
TABLE 5 | The elimination of anthraquinones.

\begin{tabular}{|c|c|c|c|c|c|}
\hline \multirow{2}{*}{$\begin{array}{l}\text { Pharmacokinetic } \\
\text { parameters }\end{array}$} & \multicolumn{2}{|c|}{$\mathrm{T}_{1 / 2}(\mathrm{~h})$} & \multicolumn{2}{|c|}{ CL L/Kg.h } & \multirow[t]{2}{*}{ References } \\
\hline & Rats & Dogs & Rats & Dogs & \\
\hline Aloe-emodin & $0.27-162.12$ & $2.02-14.73$ & $0.002-166.76$ & 61.63 & $\begin{array}{l}\text { (Feng et al., 2012; Yang et al., 2012; Li et al., 2013b: Zhang et al., 2013a; Feng } \\
\text { et al., 2014; }\end{array}$ \\
\hline Alizarin & 8.97 & - & - & - & (Gao et al., 2018) \\
\hline Aurantio-obtusin & $4.94-13.78$ & - & 1.88 & - & (Zhang et al., 2014; Yang et al., 2015; Yang et al., 2019a) \\
\hline Chrysophanol & 0.36-20.99 & $1.95-15.18$ & $0.001-44.74$ & 146.61 & $\begin{array}{l}\text { (Yang et al., 2012; Feng et al., 2013; Feng et al., 2014; Jiang et al., 2015; Zhu et al., } \\
\text { 2017) }\end{array}$ \\
\hline $\begin{array}{l}\text { Chrysophanol-8-O- } \\
\beta \text {-D-glycoside }\end{array}$ & 4.8 & - & - & - & (Ullah et al., 2018) \\
\hline Chryso-obtusin & $3.86-8.69$ & - & 3.04 & - & (Zhang et al., 2014; Yang et al., 2019a) \\
\hline Citreorosein & 3.97 & - & - & - & (Cheng et al., 2020) \\
\hline Emodin & $0.10-53.99$ & $1.72-18.73$ & $0.006-56.4$ & 17.12 & $\begin{array}{l}\text { (Song et al., 2009a:; Yang et al., 2012; Li et al., 2013b; Zhang et al., 2013a; Zhang } \\
\text { et al., 2013b; Feng et al., 2014; Zhu et al., 2014; Zhang et al., 2018c) }\end{array}$ \\
\hline Emodin-8-O- $\beta$-D-glycoside & $0.18-3.92$ & - & - & - & (Zhang et al., 2018b; Cheng et al., 2020) \\
\hline Munjistin & $9.22-11.97$ & - & - & - & (Gao et al., 2016; Gao et al., 2018) \\
\hline Obtusifolin & $1.87-11.12$ & - & 21.10 & - & (Zhang et al., 2012; Yang et al., 2015; Yang et al., 2019a) \\
\hline Obtusin & $4.41-8.28$ & - & 1.96 & - & (Zhang et al., 2014; Yang et al., 2019a) \\
\hline Physcion & $0.28-39.12$ & 13.08 & $10.10-27.35$ & 109.53 & (Feng et al., 2013; Feng et al., 2014; Feng et al., 2017; Zhu et al., 2017) \\
\hline $\begin{array}{l}\text { Physcion-8-O- } \beta-D- \\
\text { glycoside }\end{array}$ & $6.13-6.20$ & - & - & - & (Ullah et al., 2018; Cheng et al., 2020) \\
\hline Purpurin & $8.07-9.52$ & - & - & - & (Gao et al., 2016; Gao et al., 2018) \\
\hline Questinol & 8.90 & - & - & - & (Cheng et al., 2020) \\
\hline Rhein & $0.15-39.39$ & $1.8-10.11$ & $0.002-17.2$ & 0.98 & $\begin{array}{l}\text { (Yang et al., 2012; Zhang et al., 2013a; Li et al., 2013b; Zhang et al., 2013b; Feng } \\
\text { et al., 2014; Zhu et al., 2014; Zhu et al., 2017; Zhang et al., 2018c) }\end{array}$ \\
\hline Xanthopurpurin & 8.1 & - & - & - & (Han et al., 2020) \\
\hline 1-desmethylobtusin & 7.01 & - & 1.33 & - & (Zhang et al., 2014) \\
\hline
\end{tabular}

$T_{1 / 2}$ : half time of elimination; $C L$ : plasma clearance rate.

\section{Drugs}

Drug-Drug Interactions For drug compatibility, combination with Scutellariae Radix increases the urinary excretion of emodin in Rhei Radix et Rhizoma compared with oral administration of Rhei Radix et Rhizoma alone in rats $(\mathrm{Wu}$ et al., 2010; Li J. et al., 2018). Glycyrrhizae Radix et Rhizoma increases the elimination rate of rhein in Rhei Radix et Rhizoma. This may attenuate the hepatotoxicity of rhein in Rhei Radix et Rhizoma (Han et al., 2010).

The compatibility of Rhei Radix et Rhizoma and Aconiti Lateralis Radix Praeparata (Fuzi) is the basic herb pair applied in many traditional Chinese prescriptions. Studies have shown that Aconiti Lateralis Radix Praeparata decreases the clearance of aloe-emodin, chrysophanol and rhein. Therefore, the safety of the herb pair Rhei Radix et Rhizoma and Aconiti Lateralis Radix Praeparata should be given more attention (Li et al., 2015).

For the formula Dahuang-mudan decoction (DMD), in which Rhei Radix et Rhizoma is combined with Magnoliae Officinalis Cortex, Aurantii Fructus Immaturus, and Natrii Sulfas, Zhang reported that the prolonged elimination of aloe-emodin and emodin, indicating a lower toxicity in this formula. The underlying mechanisms may be due to competitive inhibition between the chemical compounds in DMD and need to be further investigated (Nong et al., 2019). An eight-herb formula NiuHuang-Jie-Du tablets (NHJDT), including Bovis Calculus (Niuhuang), Rhei Radix et Rhizoma, Realgar $\left(\mathrm{As}_{2} \mathrm{~S}_{2}\right.$, Xionghuang), Gypsum Fibrosum $\left(\mathrm{CaSO}_{4} \cdot 2 \mathrm{H}_{2} \mathrm{O}\right.$, Shigao), Platycodonis Radix (Jiegeng), and Borneolum Syntheticum (D-borneoland, Bingpian), exerts heat-clearance and detoxicification in Chinese medicine. The data showed that the clearance of chrysophanol isomers in NHJDT increased in rats, indicating that drug-drug interaction for excretion occured between the ingredients in NHJDT. However, the mechanism is still unknown (Liu Y. et al., 2018).

The elimination of anthraquinones is listed in Table 5.

\section{DISCUSSION}

Anthraquinones are naturally present in medicinal plants, especially Chinese medicines. They have attracted increasing research attention because of their pharmacological and toxicological effects. Thus, the approach to determining their PK plays a key role in exploring their actions and mechanisms. In this study, 33 out of 217 free anthraquinones and glycosides were studied for their PK (Tables 1-5 and Supplementary Table S1; Figure 1). This may result from well-investigated actions and/or detectable concentrations either in plants or in vivo for the 33 compounds. The other compounds without PK studies may be difficult to isolate from natural plants, undetectable and/or weak bioactions.

Regarding the factors influencing the PK of anthraquinones, it is suggested to consider all in vivo processes instead of absorption, distribution, metabolism or elimination alone. For example, there are multiple factors influencing the bioavailability of rhein. The differences $T_{\max }$ and AUC difference of rhein between females and males always invole complex factors, including different body weights, apparent distribution volumes and fat ratios (which are associated with absorption and distribution), phase I and phase II metabolism (other anthraquinone glycosides, sennoside A/B, aloe-emodin, can all be transformed into rhein and 
subsequently form a blood accumulation of rhein when multiple anthraquinone-containing medicinal herbs are administered) (Shia et al., 2011a; Zhang et al., 2015), and live and kidney blood flow and glomerular filtration rates (which link with the process of elimination) (Zhu et al., 2006).

In addition, with the increasing use of Chinese medicines, drugdrug interactions for anthraquinones in Chinese formulae affect all processes of PK. Even in a single herb, e.g., Polygoni Multiflori Radix (Heshouwu) (Figures 1C,F), the drug-drug interactions between the components are complicated. On the one hand, TSG inhibits UGTs and decreases the elimination of emodin to enhance the effects and toxicity of emodin (Ma et al., 2013; Yu et al., 2017). On the other hand, TSG induces the activity of CYPs and accelerates the elimination of emodin (Xing et al., 2019), which may attenuate the effects or toxicity of emodin. Our previous studies found that the anticancer efficacy of $400 \mu \mathrm{g} / \mathrm{mL}$ of ethanol extract of Polygoni Multiflori Radix (containing approximately $1.48 \mu \mathrm{M}$ of emodin) ( $\mathrm{Li} \mathrm{H}$. et al., 2018) was similar to that of $100 \mu \mathrm{M}$ emodin alone (Yang N. et al., 2019). Given the different anticancer effects of anthraquinones (Yang et al., 2018), it is strongly suggested that there would be drug interactions between ingredients in Polygoni Multiflori Radix in vivo. Actually, they are transformed each other in vivo via intestinal flora, and/or liver enzymes (Li P. et al., 2017; Xu et al., 2018; Huang et al., 2019). This may increase their efficacy and/or toxicity. Therefore, it would be very important to rationally investigate the in vivo processes of anthraquinone-containing Chinese medicines in clinical settings.

Traditoinel Chiense medicine theory facilitates preparation and formulae using drug interactions for rational drug use. These methods are very commonly used for drug processing of Chinese medicine (known as Paozhi) to induce bioavailability, enhance efficacy and/or decrease adverse drug reactions. For example, ethanol can accelerate metabolism including hydrolysis of anthraquinones glycosides in Rhei Radix et Rhizoma. Thus emodin, physcion and chrysophanol have higher bioavailability in Rhei Radix et Rhizoma steamed with wine (Zhang et al., 2019). Another interesting example of drug interactions is the ancient classic formula Rhubarb Peony decoction (Da Huang Mu dan Tang) from the Han Dynasty of China. The formula consists of five components, Rhei Radix et Rhizoma, Moutan Radix Cortex, Persicae Semen, Benincasae Semen (Dongguazi) and Natrii Sulfas, among which Natrii Sulfas can decrease the $C_{\max }$ of rhein during absorption and metabolism. This results in the diminished toxicity of rhubarb in Rhubarb Peony decoction (Zhang Y. X. et al., 2013).

It is worth noting that the metabolism of anthraquinones extends to multiple processes and is transformed into multiple products. For example, processed rhubarb aqueous extracts with rat intestinal bacteria lead to the hydrolysis of 12 anthraquinone glycosides to anthraquinone aglycones. Then, the latter are subsequently transformed to reduction and acetoxyl derivatives (Song et al., 2012). For the anthraquinone glycoside-containing formula, Xiao-Cheng-Qi decoction (XCQD) incubated with human intestinal bacteria in vitro leads to the hydrolysis of six anthraquinone glycosides to aglycones. The latter are transformed to rhein, which is further hydrolyzed to rheinanthrones (Liu X. Y. et al., 2018) (Figure 1 and Table 4).
The PK of anthraquinones may be illustrated in Figure 1.

\section{CONCLUSION}

Some anthraquinones and their glycosides, such as aloe-emodin, chrysophanol, emodin, physcion, rhein and sennosides, have attracted the most $\mathrm{PK}$ research interest due to their greater biological activities and/or detectability. Anthraquinones are mainly absorbed in the intestines and are mostly distributed in blood flow-rich tissues and organs. They may have two absorption peaks because of the hepato-intestinal circle, reabsorption in organs/tissues and glycoside hydrolysis. Drug-drug interactions influencing PK may provide insights into drug compatibility theory to enhance or reduce pharmacological/toxicological effects in Chinese medicine formulae and deserve deep investigation.

\section{DATA AVAILABILITY MATERIALS}

The datasets supporting the conclusions of this article are included within the article and its additional files.

\section{AUTHOR CONTRIBUTIONS}

XW, ML, YF and Hongliang Li designed the study; DW, XY, FC, $\mathrm{XC}$ and PC collected the data; DW and $\mathrm{X}-\mathrm{H}$. W wrote the manuscript; XW, YF and ML revised the manuscript.

\section{FUNDING}

The study was financially supported by the National Natural Science Foundation of China (8174356), the Open Project of Hubei Key Laboratory of Wudang Local Chinese Medicine Research (Hubei University of Medicine) (WDCM2018002, WDCM201917 and WDCM201918), the Chinese Medicine Project of Health Commission of Hubei Province (ZY2021010), and the Foundation for Innovative Research Team of Hubei University of Medicine (2018YHKT01). The funders played no role in the design of this study or in the collection, analysis, and interpretation of data and the writing of the manuscript, which are completely the responsibilities of the authors.

\section{ACKNOWLEDGMENTS}

The authors thank Mrs. Ming Liu, Mrs. Xiaoyan Zhang and Mr. Xuming Yu for their technical supports.

\section{SUPPLEMENTARY MATERIAL}

The Supplementary Material for this article can be found online at: https://www.frontiersin.org/articles/10.3389/fphar.2021.638993/ full\#supplementary-material. 


\section{REFERENCES}

Abu, N., Zamberi, N. R., Yeap, S. K., Nordin, N., Mohamad, N. E., Romli, M. F., et al. (2018). Subchronic toxicity, immunoregulation and anti-breast tumor effect of Nordamnacantal, an anthraquinone extracted from the stems of Morinda citrifolia L. BMC Compl. Altern. Med. 18, 31. doi:10.1186/s12906018-2102-3

Burkard, I., von Eckardstein, A., and Rentsch, K. M. (2005). Differentiated quantification of human bile acids in serum by high-performance liquid chromatography-tandem mass spectrometry. J. Chromatogr. B Anal. Technol. Biomed. Life Sci. 826, 147-159. doi:10.1016/j.jchromb.2005.08.016

Chen, M., Wei, S., Luo, C., Chen, F., Song, S., Shen, Q., et al. (2017). Simultaneous determination of wogonin, oroxylin a, schisandrin, paeoniflorin and emodin in rat serum by HPLC-MS/MS and application to pharmacokinetic studies. Biomed. Chromatogr. 31, e3966. doi:10.1002/bmc.3966

Chen, Q., He, H., Luo, S., Xiong, L., and Li, P. (2014). A novel GC-MS method for determination of chrysophanol in rat plasma and tissues: application to the pharmacokinetics, tissue distribution and plasma protein binding studies. J. Chromatogr. B Anal. Technol. Biomed. Life Sci. 973C, 76-83. doi:10.1016/ j.jchromb.2014.10.011

Chen, R. R., Liu, J., Chen, Z., Cai, W. J., Li, X. F., and Lu, C. L. (2020). Anthraquinones extract from Morinda angustifolia Roxb. Root alleviates hepatic injury induced by carbon tetrachloride through inhibition of hepatic oxidative stress. Evid. Based Compl. Alternat. Med. 20, 9861571. doi:10.1155/ 2020/9861571

Chen, Y. Y., Cao, Y. J., Tang, Y. P., Yue, S. J., and Duan, J. A. (2019). Comparative pharmacodynamic, pharmacokinetic and tissue distribution of Dahuang-Gancao decoction in normal and experimental constipation mice. Chin. J. Nat. Med. 17, 871-880. doi:10.1016/S1875-5364(19)30104-9

Cheng, W., Li, Y., Yang, W., Wu, S., Wei, M., Gao, Y., et al. (2020). Simultaneous determination of 13 constituents of Radix Polygoni Multiflori in rat plasma and its application in a pharmacokinetic study. Int. J. Anal. Chem. 2020, 4508374. doi:10.1155/2020/4508374

Cui, Y., Lu, P., Song, G., Liu, Q., Zhu, D., and Liu, X. (2016). Involvement of PI3K/ Akt, ERK and p38 signaling pathways in emodin-mediated extrinsic and intrinsic human hepatoblastoma cell apoptosis. Food Chem. Toxicol. 92, 26-37. doi:10.1016/j.fct.2016.03.013

Dai, X. Y., Yan, Y. L., Wu, Q. F., Yu, C. H., Liu, X., and Jiang, Y. Q. (2014). Comparative pharmacokinetics of rhein and chrysophanol after oral administration of Quyu Qingre granules in normal and acute blood stasis rabbits. J. Ethnopharmacol. 153, 338-343. doi:10.1016/j.jep.2014.02.005

Di, X., Wang, X., Di, X., and Liu, Y. (2015). Effect of piperine on the bioavailability and pharmacokinetics of emodin in rats. J. Pharm. Biomed. Anal. 115, 144-149. doi:10.1016/j.jpba.2015.06.027

Ding, M., Ma, S., and Liu, D. (2003). Simultaneous determination of hydroxyanthraquinones in rhubarb and experimental animal bodies by high-performance liquid chromatography. Anal. Sci. 19, 1163-1165. doi:10. 2116/analsci.19.1163

Dong, L. C., Fan, Y. X., Yu, Q., Ma, J., Dong, X., Li, P., et al. (2015). Synergistic effects of rhubarb-gardenia herb pair in cholestatic rats at pharmacodynamic and pharmacokinetic levels. J. Ethnopharmacol. 175, 67-74. doi:10.1016/j.jep. 2015.09.012

Du, D., Wu, X., Fan, C., Liang, Y., Xin, G., Zhang, R., et al. (2014). Pharmacokinetics and tissue distribution of 131 I-labeled emodin following intravenous administration to animals. Ying Yong Ji Chu Yu Gong Cheng Ke Xиe Xиe Bao 22 (1), 53-57. doi:10.3969/j.issn.1005-0930.2014.01.006

Fan, M., Peng, C., Peng, Y., Zhang, M., and Li, X. (2016). Analysis of metabolites of anthraquinones by human fecal bacteria using UPLC-Q-TOF-HRMS/MS. Chromatographia 79, 1593-1604. doi:10.1007/s10337-016-3183-0

Fang, F., Wang, J. B., Zhao, Y. L., Jin, C., Kong, W. J., Zhao, H. P., et al. (2011). A comparative study on the tissue distributions of rhubarb anthraquinones in normal and CCl4-injured rats orally administered rhubarb extract. J. Ethnopharmacol. 137, 1492-1497. doi:10.1016/j.jep.2011.08.028

Feng, S. X., Li, J. S., Qu, L. B., Shi, Y. M., and Zhao, D. (2013). Comparative pharmacokinetics of five rhubarb anthraquinones in normal and thrombotic focal cerebral ischemia-induced rats. Phytother. Res. 27, 1489-1494. doi:10. $1002 /$ ptr.4890
Feng, S. X., Li, J. S., Wang, S. M., Yang, R., Zhou, T. Q., Li, X. Y., et al. (2014). Simultaneous determination and pharmacokinetics of five rhubarb anthraquinones in dog plasma by HPLC after orally administration the rhubarb extract. Pak. J. Pharm. Sci. 27, 847-854.

Feng, S., Xu, H., Li, J., Qu, L., Yang, R., and Li, J. (2012). Determination of aloeemodin in rat plasma by SPE-HPLC and the pharmacokinetic study. Zhong Guo Xin Yao Za Zhi 21 (8), 908-911.

Feng, S., Zhang, L., Hao, R., Zhou, T., Li, X., Wang, Z., et al. (2017). Determination of physcion in plasma by SPE- HPLC and its pharmacokinetic study in rats. Ji Nan Da Xue Xue Bao 38 (2), 109-113. doi:10.11778/j.jdxb.2017.02.003

Gao, M., Wang, Z., Yang, J., Wang, L., Wu, C., Cui, B., et al. (2018). Simultaneous determination and pharmacokinetics study of four quinones in rat plasma by ultra high performance liquid chromatography with electrospray ionization tandem mass spectrometry after the oral administration of Qianzhi capsules. J. Sep. Sci. 41, 2161-2168. doi:10.1002/jssc.201700981

Gao, M., Yang, J., Wang, Z., Yang, B., Kuang, H., Liu, L., et al. (2016). Simultaneous determination of Purpurin, Munjistin and Mollugin in rat plasma by ultra high performance liquid chromatography-tandem mass spectrometry: application to a pharmacokinetic study after oral administration of Rubia cordifolia L. Extract. Mol. 21, 717. doi: $10.3390 /$ molecules 21060717

Gong, H. L., Tang, W. F., Wang, H., Xia, Q., and Huang, X. (2011). Effects of food and gender on the pharmacokinetics of rhein and emodin in rats after oral dosing with Da-Cheng-Qi decoction. Phytother. Res. 25, 74-80. doi:10.1002/ptr. 3223

Gong, H. L., Tang, W. F., Yu, Q., Xiang, J., Xia, Q., Chen, G. Y., et al. (2009). Effect of severe acute pancreatitis on pharmacokinetics of Da-Cheng-Qi Decoction components. World J. Gastroenterol. 15, 5992-5999. doi:10.3748/ wjg.15.5992

Gong, X. H., Li, Y., Zhang, R. Q., Xie, X. F., Peng, C., and Li, Y. X. (2015). The synergism mechanism of Rhubarb Anthraquinones on constipation elucidated by comparative pharmacokinetics of Rhubarb extract between normal and diseased rats. Eur. J. Drug Metab. Pharmacokinet. 40, 379-388. doi:10.1007/ s13318-014-0216-7

Guo, R., Wu, H., Yu, X., Xu, M., Zhang, X., Tang, L., et al. (2017). Simultaneous determination of seven anthraquinone aglycones of crude and processed semen cassiae extracts in rat plasma by UPLC-MS/MS and its application to a comparative pharmacokinetic study. Molecules 22, 1803. doi:10.3390/ molecules 22111803

Han, D. E., Shi, Y., Tian, P., Wei, H., Miao, M., and Li, X. M. (2020). Development of an ultra-performance liquid chromatography-electrospray ionizationOrbitrap mass spectrometry method for determination of xanthopurpurin in rat plasma and its application to pharmacokinetic study. Biomed. Chromatogr. 34, e4838. doi:10.1002/bmc.4838

Han, G., Kang, X., Zhai, G., Fan, Y., Wang, Y., Liu, L., et al. (2010). Effect of compatibility using Glycyrrhiza and rhubarb on pharmacokinetics of rhein in rats. Zhong Guo Shi Yan Fang Ji Xue Za Zhi 16 (9), 72-74. doi:10.13422/j.cnki. syfjx.2010.09.060

He, L. N., Yang, A., Cui, T., Zhai, Y., Zhang, F., Chen, J., et al. (2015). Reactive metabolite activation by CYP2C19-mediated rhein hepatotoxicity. Xenobiotica 45, 361-372. doi:10.3109/00498254.2014.984794

Hou, M. L., Chang, L. W., Lin, C. H., Lin, L. C., and Tsai, T. H. (2014). Determination of bioactive components in Chinese herbal formulae and pharmacokinetics of rhein in rats by UPLC-MS/MS. Molecules 19, 4058-4075. doi:10.3390/molecules19044058

Huang, J., Yan, X., and Feng, F. (2014). Simultaneous determination of four anthraquinones in rat plasma by HPLC-FLD method and its pharmacokinetic study. Zhong Guo Zhong Yao Za Zhi 39, 3850-3854. doi:10.4268/ cjcmm 20141937

Huang, J., Zhang, J. P., Bai, J. Q., Wei, M. J., Zhang, J., Huang, Z. H., et al. (2018). Chemical profiles and metabolite study of raw and processed Polygoni Multiflori Radix in rats by UPLC-LTQ-Orbitrap MSn spectrometry. Chin. J. Nat. Med. 16, 375-400. doi:10.1016/S1875-5364(18)30070-0

Huang, Z., Xu, Y., Wang, Q., and Gao, X. (2019). Metabolism and mutual biotransformations of anthraquinones and anthrones in rhubarb by human intestinal flora using UPLC-Q-TOF/MS. J. Chromatogr. B Analyt. Technol. Biomed. Life Sci. 1104, 59-66. doi:10.1016/j.jchromb.2018.10.008

Jiang, L., Yu, L., Zhang, Q., Shang, G., Yu, W., Shan, S., et al. (2015). In vivo pharmacokinetic study on several active components of Dachengqi Decoction 
in rats by UPLC-MS/MS. Zhong Cao Yao 46 (19), 2908-2915. doi:10.7501/j. issn.0253-2670.2015.19.015

Kong, W., Xia, X., and Wang, J. (2011). Solid-phase extraction and ultra highperformance liquid chromatography tandem mass spectrometry analysis of the gastrointestinal absorption of emodin in different digestive segments of rats. J. Sep. Sci. 34, 260-267. doi:10.1002/jssc.201000757

Koyama, J., Takeuchi, A., Morita, I., Nishino, Y., Shimizu, M., Inoue, M., et al. (2009). Characterization of emodin metabolites in Raji cells by LC-APCI-MS/ MS. Bioorg. Med. Chem. 17, 7493-7499. doi:10.1016/j.bmc.2009.09.024

Lang, W. (1993). Pharmacokinetic-metabolic studies with 14C-aloe emodin after oral administration to male and female rats. Pharmacology 47, 110-119. doi:10. $1159 / 000139849$

Li, C., Ma, J., and Li, H. (2012). Research Progress on absorption and metabolism of anthraquinones. Yao Wu Sheng Wu Ji Shu 19, 557-560. doi:10.1016/01637827(89)90012-x

Li, D., Zhang, N., Cao, Y., Zhang, W., Su, G., Sun, Y., et al. (2013a). Emodin ameliorates lipopolysaccharide-induced mastitis in mice by inhibiting activation of NF-kB and MAPKs signal pathways. Eur. J. Pharmacol. 705, 79-85. doi:10.1016/j.ejphar.2013.02.021

Li, H., Cao, S., Wang, X., Zuo, Q., Chen, P., Liu, Y., et al. (2016). Quality evaluation of Heshouwu, a taoist medicine in Wudang, China. Exp. Ther. Med. 12, 2317-2323. doi:10.3892/etm.2016.3580

Li, H., Guo, H., Wu, L., Zhang, Y., Chen, J., Liu, X., et al. (2013b). Comparative pharmacokinetics study of three anthraquinones in rat plasma after oral administration of Radix et Rhei Rhizoma extract and Dahuang Fuzi Tang by high performance liquid chromatography-mass spectrometry. J. Pharm. Biomed. Anal. 76, 215-218. doi:10.1016/j.jpba.2012.12.004

Li, H., Wang, X., Liu, Y., Pan, D., Wang, Y., Yang, N., et al. (2017a). Hepatoprotection and hepatotoxicity of Heshouwu, a Chinese medicinal herb: context of the paradoxical effect. Food Chem. Toxicol. 108 (Pt B), 407-418. doi:10.1016/j.fct.2016.07.035

Li, H., Xiang, L., Yang, N., Cao, F., Li, C., Chen, P., et al. (2018a). Zhiheshouwu ethanol extract induces intrinsic apoptosis and reduces unsaturated fatty acids via SREBP1 pathway in hepatocellular carcinoma cells. Food Chem. Toxicol. 119, 169-175. doi:10.1016/j.fct.2018.04.054

Li, H., Zhang, Y., Yu, Y., Wang, P., Li, F., and Meng, X. (2013c). Pharmacokinetic/ pharmacodynamic modeling of antipyretic and reducing plasma concentration of NO effects of Rheum palmatum in rat. Zhong Guo Zhong Yao Za Zhi 38, 1231-1236.

Li, J., Lv, Z., Gao, K., and Liu, J. (2018b). Analysis of blood and urine components of rats after oral administration of Scutellariae Radix, Rhei Radix et rhizoma and their compatibility. Yin Shi Bao Jian 14, 70. doi:10. $4268 / \mathrm{cjcmm} 20120410$

Li, P., Lu, Q., Jiang, W., Pei, X., Sun, Y., Hao, H., et al. (2017b). Pharmacokinetics and pharmacodynamics of rhubarb anthraquinones extract in normal and disease rats. Biomed. Pharmacother. 91, 425-435. doi:10.1016/j.biopha.2017. 04.109

Li, S., and Feng, S. (2018). Studies on pharmacokinetics and the tissue distribution of aloe-emodin in mice. Zhong Yao Yao Li Yu Lin Chuang 34 (6), 34-38. doi:10. 13412/j.cnki.zyyl.2018.06.010

Li, X., Ma, W., Guo, B., and Zeng, Z. (2003). Analysis of anthraquinones in serum and urine after oral administration of semen cassiae. Zhong Guo Zhong Yao Za Zhi 28 (9), 59-61. doi:10.4268/cjcmm20151319

Li, Y. X., Gong, X. H., Li, Y., Zhang, R. Q., Yuan, A., Zhao, M. J., et al. (2015). The influence of aconitum carmichaelii Debx. On the pharmacokinetic characteristics of main components in Rheum Palmatum L. Phytother. Res. 29, 1259-1264. doi:10.1002/ptr.5369

Lin, L., Ni, B., Lin, H., Zhang, M., Li, X., Yin, X., et al. (2015). Traditional usages, botany, phytochemistry, pharmacology and toxicology of Polygonum multiflorum Thunb.: a review. J. Ethnopharmacol. 159, 158-183. doi:10. 1016/j.jep.2014.11.009

Liu, W., Tang, L., Ye, L., Cai, Z., Xia, B., Zhang, J., et al. (2010). Species and gender differences affect the metabolism of emodin via glucuronidation. AAPS. J. 12, 424-436. doi:10.1208/s12248-010-9200-6

Liu, W., Zheng, Z., Liu, X., Gao, S., Ye, L., Yang, Z., et al. (2011a). Sensitive and robust UPLC-MS/MS method to determine the gender-dependent pharmacokinetics in rats of emodin and its glucuronide. J. Pharm. Biomed. Anal. 54, 1157-1162. doi:10.1016/j.jpba.2010.12.004
Liu, X., Cui, Y., Chen, D., Li, Z., Chang, J., and Liu, C. (2011b). Study on in situ rats stomach and intestinal absorption of the total anthraquinones of rhubarb. Zhong Guo Yi Yuan Yao Xue Za Zhi 31, 188-191. doi:10.4268/cjcmm20140228

Liu, X., Li, H., Wu, L., Xing, J., Poh, Y., Cai, H., et al. (2015). Simultaneous quantification of chrysophanol and physcion in rat plasma by ultra fast liquid chromatography-tandem mass spectrometry and application of the technique to comparative pharmacokinetic studies of Radix et Rhei Rhizoma extract alone and Dahuang Fuzi Decoction. J. Chromatogr. B. Analyt. Technol. Biomed. Life Sci. 980, 88-93. doi:10.1016/j.jchromb.2014.11.025

Liu, X. Y., Li, L., Li, X. Q., Yu, B. Y., and Liu, J. H. (2018a). Identification of active compound combination contributing to anti-inflammatory activity of XiaoCheng-Qi Decoction via human intestinal bacterial metabolism. Chin. J. Nat. Med. 16, 513-524. doi:10.1016/S1875-5364(18)30088-8

Liu, Y., Wu, X., Guan, R., Hang, T., and Song, M. (2018b). Comparative pharmacokinetics of active anthraquinones ingredients after oral administration of Rhei Radix et Rhizoma and Niuhuang Jiedu Tablets to rats. Zhong Guo Yao Ke Da Xue Xue Bao 49 (4), 449-455. doi:10.11665/j. issn.1000-5048.20180410

Luo, L., Schomaker, S., Houle, C., Aubrecht, J., and Colangelo, J. L. (2014). Evaluation of serum bile acid profiles as biomarkers of liver injury in rodents. Toxicol. Sci. 137, 12-25. doi:10.1093/toxsci/kft221

Ma, J., Zheng, L., Deng, T., Li, C. L., He, Y. S., Li, H. J., et al. (2013). Stilbene glucoside inhibits the glucuronidation of emodin in rats through the downregulation of UDP-glucuronosyltransferases 1A8: application to a drug-drug interaction study in Radix Polygoni Multiflori. J. Ethnopharmacol. 147, 335-340. doi:10.1016/j.jep.2013.03.013

Ma, J., Zheng, L., He, Y. S., and Li, H. J. (2015). Hepatotoxic assessment of Polygoni Multiflori Radix extract and toxicokinetic study of stilbene glucoside and anthraquinones in rats. J. Ethnopharmacol. 162, 61-68. doi:10.1016/j.jep. 2014.12.045

Ma, Y., Zhao, Y., Xie, H., Wang, T., Zhu, S., Wu, Y., et al. (2005). Pharmacokinetics of anthraquinones in rats after intragastric gavage of taohechengqi decoction. Zhong Guo Yao Li Xue Tong Bao (10), 124-127.

Matsui, E., Takayama, K., Sato, E., and Okamura, N. (2011). The influence of glycyrrhiza and antibiotics on the purgative action of sennoside a from Daiokanzoto in mice. Biol. Pharm. Bull. 34, 1438-1442. doi:10.1248/bpb.34. 1438

Meng, Z., and Ding, X. (2019). Recent advances in the study of Polygoni Multiflori Radix: its absorbed components in rat blood, pharmacokinetics and Metalobomic study of hepatotoxicity. Yao Xue Jin Zhan 43 (5), 379-383. doi:10.26226/morressier.57d6b2bbd462b8028d88ed04

Nong, F., Zhao, Z., Luo, X., Liu, C., Li, H., Liu, Q., et al. (2019). Evaluation of the influence of mirabilite on the absorption and pharmacokinetics of the ingredients in Dahuang-mudan decoction by a validated UPLC/QTOF-MS/ MS method. Biomed. Chromatogr. 33, e4423. doi:10.1002/bmc. 4423

Qin, X., Peng, Y., and Zheng, J. (2018). In Vitro and in Vivo studies of the electrophilicity of physcion and its oxidative metabolites. Chem. Res. Toxicol. 31, 340-349. doi:10.1021/acs.chemrestox.8b00026

Qiu, Y., Wang, P., Wang, J., Zhang, Y., and Meng, X. (2011). Research on intestinal absorption of rhein in the rats. Zhong Yao Yao Li Yu Lin Chuang 27 (5), 31-34. doi:10.13412/j.cnki.zyyl.2011.05.012

Ren, Y., Gong, H., Tang, W., Wan, M., and Huang, X. (2009). Effects of ranitidine on pharmacokinetics of rhein from Dachengqi Decoction in rats after oral administration. Zhong Xi Yi Jie He Xue Bao 7 (9), 868-872. doi:10.3736/ jcim20090913

Rong, Y., Feng, S., Wu, C., Liu, D., and Wang, S. (2016). Research progress of pharmacokinetics and metabolism of chemical compositions in Rhei radix et Rhizoma. Zhong Guo Shi Yan Fang Ji Xue Za Zhi 22, 199-203. doi:10.13422/j. cnki.syfjx.2016100199

Shao, M., and Feng, F. (2015). Comparative pharmacokinetics of anthraquinones from Zhi-Zi-Da-Huang decoction in normal and alcohol-induced hepatic injury rats. Guang Zhou Hua Gong 43 (6), 53-56. doi:10.1016/j.jep.2009.08.028

Shia, C., Hou, Y., Tsai, S., Huieh, P., Leu, Y., and Chao, P. L. (2010). Differences in pharmacokinetics and ex vivo antioxidant activity following intravenous and oral administrations of emodin to rats. J. Pharm. Sci. 99, 2185-2195. doi:10. $1002 /$ jps. 21978

Shia, C. S., Hou, Y., Juang, S., Tsai, S., Hsieh, P., Ho, L., et al. (2011a). Metabolism and pharmacokinetics of San-Huang-Xie-Xin-tang, a Polyphenol-rich Chinese 
medicine formula, in rats and ex-vivo antioxidant activity. Evid. Based Complement Alternat. Med. 2011, 721293. doi:10.1093/ecam/nep124

Shia, C. S., Juang, S. H., Tsai, S. Y., Chang, P. H., and Chao, P. D. L. (2009). Metabolism and pharmacokinetics of anthraquinones in Rheum palmatum in rats and ex vivo antioxidant activity. Planta. Med. 75, 1386-1392. doi:10.1055/s0029-1185725

Shia, C. S., Tsai, S. Y., Lin, J. C., Li, M. L., Ko, M. H., Chao, P. D., et al. (2011b). Steady-state pharmacokinetics and tissue distribution of anthraquinones of Rhei Rhizoma in rats. J. Ethnopharmacol. 137, 1388-1394. doi:10.1016/j.jep. 2011.08.003

Solárová, Z., Liskova, A., Samec, M., Kubatka, P., Büsselberg, D., and Solár, P. (2020). Anticancer potential of lichens' secondary metabolites. Biomolecules 10, 87. doi:10.3390/biom 10010087

Song, R., Lin, H., Zhang, Z., Li, Z., Xu, L., Dong, H., et al. (2009a). Profiling the metabolic differences of anthraquinone derivatives using liquid chromatography/tandem mass spectrometry with data-dependent acquisition. Rapid Commun. Mass Spectrom. 23, 537-547. doi:10.1002/rcm. 3907

Song, R., Tian, Y., and Zhang, Z. (2012). Comparison of transformation of four processed rhubarb aqueous extracts in intestinal bacteria in vitro. Zhong Guo Zhong Yao Za Zhi 37 (12), 1755-1760. doi:10.4268/cjcmm20121214

Song, R., Xu, F., Zhang, Z., Liu, Y., Dong, H., and Tian, Y. (2008). Structural elucidation of in vitro metabolites of emodin by liquid chromatography-tandem mass spectrometry. Biomed. Chromatogr. 22, 1230-1236. doi:10.1002/bmc. 1050

Song, R., Xu, L., Xu, F., Dong, H., Tian, Y., and Zhang, Z. (2011). Metabolic analysis of rhubarb extract by rat intestinal bacteria using liquid chromatographytandem mass spectrometry. Biomed. Chromatogr. 25, 417-426. doi:10.1002/ bmc. 1467

Song, R., Xu, L., Xu, F., Li, Z., Dong, H., Tian, Y., et al. (2010). In vivo metabolism study of rhubarb decoction in rat using high-performance liquid chromatography with UV photodiode-array and mass-spectrometric detection: a strategy for systematic analysis of metabolites from traditional Chinese medicines in biological samples. J. Chromatogr A. 1217, 7144-7152. doi:10.1016/j.chroma.2010.09.028

Song, Z., Peng, J., and Ma, C. (2009b). LC-MS/MS determination of plasma and brain concentration of emodin in rats. Yao Wu Fen Xi Za Zhi 29, 926-930. doi:10.4268/cjcmm20141033

Sun, Y., Li, Q., and Chen, Q. (1986). Quantitative analysis of emodin and its metabolites excreted in mice. Nan Jing Yao Xue Yuan Xue Bao 17 (2), 132-135.

Sun, Y., Xin, X., Zhang, K., Cui, T., Peng, Y., and Zheng, J. (2018). Cytochrome P450 mediated metabolic activation of chrysophanol. Chem. Biol. Interact. 289, 57-67. doi:10.1016/j.cbi.2018.04.015

Takayama, K., Tsutsumi, H., Ishizu, T., and Okamura, N. (2012). The influence of rhein 8-O- $\beta$-D-glucopyranoside on the purgative action of sennoside A from rhubarb in mice. Biol. Pharm. Bull. 35, 2204-2208. doi:10.1248/bpb.b12-00632

Tan, X., Tian, J., Xin, X., Wang, S., and An, F. (2013). Isolation and purification of chrysophanol and its pharmacokinetics and tissue distribution in rabbits. Zhong Guo Xin Yao Yu Lin Chuang Za Zhi 32 (7), 555-560. doi:10.1007/bf01070902

Teng, Z. H., Zhou, S. Y., Yang, R. T., Liu, X. Y., Liu, R. W., Yang, X., et al. (2007). Quantitation assay for absorption and first-pass metabolism of emodin in isolated rat small intestine using liquid chromatography-tandem mass spectrometry. Biol. Pharm. Bull. 30, 1628-1633. doi:10.1248/bpb.30.1628

Tian, J., Chen, X., and Bai, X. (2012). Analysis of emodin and its metabolites based on hollow fiber liquid phase microextraction. Se Pu 30, 507-514. doi:10.3724/ sp.j.1123.2011.12001

Ullah, H., Kim, J., Rehman, N. U., Kim, H. J., Ahn, M. J., and Chung, H. J. (2018). A Simple and Sensitive liquid chromatography with tandem mass spectrometric method for the simultaneous determination of anthraquinone glycosides and their aglycones in rat plasma: application to a pharmacokinetic study of Rumex acetosa extract. Pharmaceutics 10, 121. doi:10.3390/pharmaceutics10030100

Wan, P., Sun, J., Hao, G., Zhang, X., Xiao, D., Liu, Z., et al. (2013). Determination of rhein in plasma, urine and feces by HPLC-fluorescence detection and its pharmacokinetics in healthy volunteers. Zhong Guo Yao Ke Da Xue Xue Bao 44 (1), 73-76. doi:10.3109/13880209.2013.805790

Wang, D., Shi, Q., and Feng, F. (2017a). Rapid discovery and identification of the prototypes and their metabolites of Da-Huang-Xiao-Shi decoction in rat plasma by an integrative strategy based on liquid chromatography coupled with mass spectrometry. J. Sep. Sci. 40, 2722-2731. doi:10.1002/jssc.201601419

Wang, J., Wang, P., Yang, Y., Meng, X., and Zhang, Y. (2011a). Intestinal absorption of aloe-emodin using single-passintestinal perfusion method in rat. Zhong Guo Zhong Yao Za Zhi 36 (17), 2393-2398. doi:10.4268/ cjcmm20111719

Wang, J., Zhao, H., Kong, W., Jin, C., Zhao, Y., Qu, Y., et al. (2010). Microcalorimetric assay on the antimicrobial property of five hydroxyanthraquinone derivatives in rhubarb. Rheum Palmatum $L$. Bifidobacterium Adolescentis. Phytomedicine 17, 684-689. doi:10.1016/j. phymed.2009.10.009

Wang, J., Zhao, Y., Xiao, X., Li, H., Zhao, H., Zhang, P., et al. (2009). Assessment of the renal protection and hepatotoxicity of rhubarb extract in rats. J. Ethnopharmacol. 124, 18-25. doi:10.1016/j.jep.2009.04.018

Wang, L., Sang, M., Liu, E., Banahene, P. O., Zhang, Y., Wang, T., et al. (2017b). Rapid profiling and pharmacokinetic studies of major compounds in crude extract from Polygonum multiflorum by UHPLC-Q-TOF-MS and UPLC-MS/ MS. J. Pharm. Biomed. Anal. 140, 45-61. doi:10.1016/j.jpba.2017.03.016

Wang, P., Meng, X., Wang, J., Liu, H., Yang, Y., and Liu, R. (2011b). Intestinal absorption kinetics of rhubarb Mixture free anthraquinones in rats. Shi Zhen Guo Yi Guo Yao 22 (4), 790-792.

Wang, P., Zheng, W., Liu, X., Zhao, D., Shu, S., and Feng, S. (2020). Studies on pharmacokinetics and tissue distribution of rhubarb anthraquinones in mice. Zhong Yao Yao Li Yu Lin Chuang 36, 85-94. doi:10.5246/jcps.2017.02.010

Wang, W., He, Y., Lin, P., Li, Y., Sun, R., Gu, W., et al. (2014). In vitro effects of active components of Polygonum Multiflorum Radix on enzymes involved in the lipid metabolism. J. Ethnopharmacol. 153, 763-770. doi:10.1016/j.jep.2014. 03.042

Wu, B., Liu, S., Sun, Z., Tang, Y., and Huang, C. (2010). Analysis of plasmic and urinary constituents after oral administration of Rheum palmatum, scutellaria baicalensis and their compatibility in rats. Shi Jie Ke Xue Ji Shu (Zhong Yi Yao Xian Dai Hua) 12, 652-656. doi:10.3969/j.issn.1674-3849.2010.04.0344

Wu, J., Ma, Y., and Yan, D. (2008). Urinary pharmacokinetics of five anthraquinones after oral administration of Xiexin Decoction in rats. Zhong Cheng Yao Za Zhi 30 (12), 1736-1740. doi:10.1002/ptr.2622

Wu, W., Hu, N., Zhang, Q., Li, Y., Li, P., Yan, R., et al. (2014). In vitro glucuronidation of five rhubarb anthraquinones by intestinal and liver microsomes from humans and rats. Chem. Biol. Interact. 219, 18-27. doi:10. 1016/j.cbi.2014.05.006

Wu, W. J., Yan, R., Li, T., Li, Y. P., Zhou, R. N., and Wang, Y. T. (2017a). Pharmacokinetic alterations of rhubarb anthraquinones in experimental colitis induced by dextran sulfate sodium in the rat. J. Ethnopharmacol. 198, 600-607. doi:10.1016/j.jep.2017.01.049

Wu, Y., Zhang, Y., Jiang, S., Chen, G., and Jiang, X. (2017b). 25, effects of wine processed Rheum palmatum on the pharmacokinetics of aloe-emodin, rhein and emodin in rats by high performance liquid chromatography/mass spectrometry. Yao Xue Yu Lin Chuang Yan Jiu 25, 392-396. doi:10.13664/j. cnki.pcr.2017.05.006

Xie, H., Ma, Y., Wang, T., and Ye, F. (2005). Pharmacokinetics of rhein in taohe chengqi decoction and rhubarb in rabbits. Zhong Yao Yao Li Yu Lin Chuang (2), 1-3. doi:10.1016/s0895-7061(99)80248-9

Xin, Y., Geng, H., Zhang, S., Liu, Z., and Ma, Y. (2009). Pharmacokinetic Study of Rhein from Sanhuang Xiexin Decoction and Radix et Rhizoma Rhei in Rats. Zhong Guo Shi Yan Fang Ji Xue Za Zhi 15 (3), 56-59. doi:10.13422/j.cnki.syfjx. 2009.03.019

Xing, Y., Wang, L., Wang, C., Zhang, Y., Zhang, Y., Hu, L., et al. (2019). Pharmacokinetic studies unveiled the drug-drug interaction between trans2,3,5, $4^{\prime}$-tetrahydroxystilbene-2-O- $\beta$-d-glucopyranoside and emodin that may contribute to the idiosyncratic hepatotoxicity of Polygoni Multiflori Radix. J. Pharm. Biomed. Anal. 164, 672-680. doi:10.1016/j.jpba.2018.11.034

$\mathrm{Xu}, \mathrm{Y}$., Wang, Q., Yin, Z., and Gao, X. (2018). On-line incubation and real-time detection by ultra-performance liquid chromatography-quadrupole time-offlight mass spectrometry for rapidly analyzing metabolites of anthraquinones in rat liver microsomes. J. Chromatogr. A. 1571, 94-106. doi:10.1016/j.chroma. 2018.08.006

Yan, Y., and Dai, X. (2014). Total quantity statistical moment analysis on pharmacokinetics of rhein and chrysophanol after oral administration of 
Quyu Qingre granules in normal and acute blood stasis rabbits. Zhong Guo Zhong Yao Za Zhi 39, 520-525. doi:10.4268/cjcmm20140331

Yang, B., Xie, L., Peng, S., Sun, K., Jin, J., Zhen, Y., et al. (2019a). Nine components pharmacokinetic study of rat plasma after oral administration raw and prepared Semen Cassiae in normal and acute liver injury rats. J. Sep. Sci. 42, 2341-2350. doi:10.1002/jssc.201900007

Yang, C., Wang, S., Guo, X., Sun, J., Liu, L., and Wu, L. (2015). Simultaneous determination of seven anthraquinones in rat plasma by Ultra High Performance Liquid Chromatography-tandem Mass Spectrometry and pharmacokinetic study after oral administration of Semen Cassiae extract. J..Ethnopharmacol. 169, 305-313. doi:10.1016/j.jep.2015.04.008

Yang, N., Cao, F., Huo, J., Li, H., Li, C., Wang, Q., et al. (2018). SREBP1-based active component screening of anthraquinones Radix Polygoni Multiflori Preparata for lowering Lipid metabolism in hepatocellular carcinoma cells. Hu Bei Yi Yao Xue Yuan Xue Bao 37, 156-160. doi:10.13819/j.issn.1006-9674. 2018.02.014

Yang, N., Li, C., Li, H., Liu, M., Cai, X., Cao, F., et al. (2019b). Emodin induced SREBP1-dependent and SREBP1-independent apoptosis in hepatocellular carcinoma cells. Front. Pharmacol. 10, 709. doi:10.3389/fphar.2019.00709

Yang, N., Xiang, L., Cao, F., Li, H., and Wang, X. (2016). Research Progress on the effects and mechanisms of emodin in the tumor Metastasis. Zhong Liu Yao Xue 6 (3), 173-177. doi:10.3969/j.issn.2095-1264.2016.03.03

Yang, Y.-M., Wang, P., Zhang, Y., Meng, X., Chen, Y., and Qiu, Y. (2012). A Comparative study on the absorption kinetics parameters of Rhubarb free anthraquinones between normal dogs and dogs with severe acute pancreatitis. Zhong Guo Zhong Xi Yi Jie He Za Zhi 32 (4), 494-498. doi:10.4268/ cjcmm20140228

Yang, Y., Wang, P., Miu, S., Meng, X., and Liu, H. (2010). Study on portal vein absorption kinetics model and rhubarb anthraquinones absorption kinetics in normal rats. Zhong Yao Yu Lin Chuang 1 (3), 39-42. doi:10.4268/ cjcmm20130931

Yu, C. P., Shia, C. S., Lin, H. J., Hsieh, Y. W., Lin, S. P., and Hou, Y. C. (2016). Analysis of the pharmacokinetics and metabolism of aloe-emodin following intravenous and oral administrations in rats. Biomed. Chromatogr. 30 (10), 1641-1647. doi:10.1002/bmc.3735

Yu, J., Guo, X., Zhang, Q., Peng, Y., and Zheng, J. (2018). Metabolite profile analysis and pharmacokinetic study of emodin, baicalin and geniposide in rats. Xenobiotica 48, 927-937. doi:10.1080/00498254.2017.1382748

Yu, Q., Jiang, L. L., Luo, N., Fan, Y. X., Ma, J., Li, P., et al. (2017). Enhanced absorption and inhibited metabolism of emodin by 2, 3, 5, 4'tetrahydroxystilbene-2-O- $\beta$-D-glucopyranoside: Possible mechanisms for Polygoni Multiflori Radix-induced liver injury. Chin. J. Nat. Med. 15, 451-457. doi:10.1016/S1875-5364(17)30067-5

Zhang, J., Fu, Y., Li, L., Liu, Y., Zhang, C., Yu, D., et al. (2019). Pharmacokinetic comparisons of major bioactive components after oral administration of raw and steamed rhubarb by UPLC-MS/MS. J. Pharm. Biomed. Anal. 171, 43-51. doi:10.1016/j.jpba.2019.04.002

Zhang, J., Wang, T., Ren, Z., Gong, L., Huang, J., Bai, J., et al. (2018a). Application of HPLC-LTQ Orbitrap MS for metabolic profiles of Polygonum multiflora extract in rats. Biomed. Chromatogr. 32. doi:10.1002/bmc.4067

Zhang, J., Zhang, J., Wang, G., Xie, H., Gu, Y., Jia, Y., et al. (2010). Pharmacokinetic comparision in absorption of Rhein in SD rats and Beagle dogs. Zhong Guo Lin Chuang Yao Li Xue Yu Zhi Liao Xue 15 (5), 511-518. doi:10.1080/00498254. 2017.1367441

Zhang, M., Lin, L., Lin, H., Qu, C., Yan, L., and Ni, J. (2018b). Interpretation the hepatotoxicity based on pharmacokinetics investigated through oral administrated different extraction Parts of Polygonum multiflorum on rats. Front. Pharmacol. 9, 505. doi:10.3389/fphar.2018.00505

Zhang, Q., Ma, Y. M., Wang, Z. T., and Wang, C. H. (2013a). Differences in pharmacokinetics and anti-inflammatory effects between decoction and maceration of Sanhuang Xiexin Tang in rats and mice. Planta. Med. 79, 1666-1673. doi:10.1055/s-0033-1350959

Zhang, W. D., Wang, P. Y., Wang, Y., Wang, Q., Gu, Y., Cao, J., et al. (2014). Simultaneous determination of aurantio-obtusin, chrysoobtusin, obtusin and 1desmethylobtusin in rat plasma by UHPLC-MS/MS. Biomed. Chromatogr. 28, 369-374. doi:10.1002/bmc.3030
Zhang, Y., Wu, X., and Gao, W. (2012). Pharmacokinetics of anthraquinones in rat plasma after oral administration of Cassia obtusifolia extract determined by HPLC method. Zhong Guo Yi Yuan Yao Xue Za Zhi 32 (9), 691-694. doi:10. 13286/j.cnki.chinhosppharmacyj.2012.09.027

Zhang, Y. X., Li, J. S., Peng, W. W., Liu, X., Yang, G. M., Chen, L. H., et al. (2013b). Comparative pharmacokinetics of aloe-emodin, rhein and emodin determined by liquid chromatography-mass spectrometry after oral administration of a rhubarb peony decoction and rhubarb extract to rats. Pharmazie 68 (5), 333-339. doi:10.1080/10826076.2011.572211

Zhang, Y., Xu, Y., Hu, Y., Wang, P., and Meng, X. (2015). Study on pharmacokinetics of rhein in acute hepatic injury rats after oral administration of rhubarb extracts. Hua Xi Yao Xue Za Zhi 30 (5), 571-574. doi:10.13375/j.cnki.wcjps.2015.05.016

Zhang, Y., Zhang, Z., and Song, R. (2018c). The influence of compatibility of rhubarb and Radix Scutellariae on the pharmacokinetics of anthraquinones and flavonoids in rat plasma. Eur. J. Drug Metab. Pharmacokinet. 43 (3), 291-300. doi:10.1007/s13318-017-0444-8

Zhao, F., Wang, S. J., Lin, S., Zhu, C. G., Yuan, S. P., Ding, X. Y., et al. (2011). Anthraquinones from the roots of Knoxia valerianoides. J. Asian. Nat. Prod. Res. 13, 1023-1029. doi:10.1080/10286020.2011.606813

Zhao, X., Yumei, Z., Juan, L., Meihua, W., Shifeng, Z., Hui, G., et al. (2015). Tissue pharmacology of Da-cheng-Qi decoction in experimental acute pancreatitis in rats. Evid Based Complement Alternat Med, 283175. doi:10.1155/2015/283175

Zhou, C., Yao, Y., Zhng, B., Pei, Q., and Jia, S. (2010). In Vivo pharmacokinetics comparison of emodin from Sanhuang tablets and extracts of crude drug rhubarb in rats. Shi Yong Yu Fang Yi Xue 17 (6), 1193-1195. doi:10.1007/ s13318-014-0216-7

Zhu, C., Zhang, D., Song, J., Song, Z., and Xue, G. (2012). Tissue distribution of three chrysophanol formulations in mice. Zhong Guo Yao Xue Za Zhi 47 (11), 898-902. doi:10.2172/7224295

Zhu, H., Bi, K., Han, F., Guan, J., Tang, Z., Chen, K., et al. (2014). Simultaneous determination of two iridoid glycosides, two anthraquinones and four flavonoid glycosides of Zhi-Zi-Da-Huang decoction in rat plasma by UFLC-MS/MS: application to a comparative pharmacokinetic study in normal and cholestatic liver injury rats. J. Chromatogr. B Analyt. Technol. Biomed. Life Sci. 960, 116-125. doi:10.1016/j.jchromb.2014.04.031

Zhu, H., Bi, K., Han, F., Guan, J., Zhang, X., Mao, X., et al. (2016). Comparison on pharmacokinetics of active constituents from Zhizi Dahuang Tang in normal and alcoholic liver injury rats. Zhong Guo Shi Yan Fang Ji Xue Za Zhi 22 (22), 77-81. doi:10.13422/j.cnki.syfjx.2016220077

Zhu, H., Bi, K., Han, F., Guan, J., Zhang, X., Mao, X., et al. (2015). Identification of the absorbed components and metabolites of Zhi-Zi-Da-Huang decoction in rat plasma by ultra-high performance liquid chromatography coupled with quadrupole-time-of-flight mass spectrometry. J. Pharm. Biomed. Anal. 111, 277-287. doi:10.1016/j.jpba.2015.03.043

Zhu, H., Liu, X., Zhu, T. T., Wang, X. L., Qin, K. M., Pei, K., et al. (2017). UHPLCMS/MS method for the simultaneous quantitation of five anthraquinones and gallic acid in rat plasma after oral administration of prepared rhubarb decoction and its application to a pharmacokinetic study in normal and acute blood stasis rats. J. Sep. Sci. 40, 2382-2389. doi:10.1002/jssc.201700166

Zhu, W., Ruan, X., and Chen, K. (2006). Effect of gender difference on pharmacokinetics of rhein healthy volunteers after oral administration of rhubarb extract. Zhong Guo Lin Chuang Yao Li Xue Yu Zhi Liao Xue 11 (2), 223-227. doi:10.1142/s0192415x05003508

Conflict of Interest: The authors declare that the research was conducted in the absence of any commercial or financial relationships that could be construed as a potential conflict of interest.

Copyright $\odot 2021$ Wang, Wang, Yu, Cao, Cai, Chen, Li, Feng, Li and Wang. This is an open-access article distributed under the terms of the Creative Commons Attribution License (CC BY). The use, distribution or reproduction in other forums is permitted, provided the original author(s) and the copyright owner(s) are credited and that the original publication in this journal is cited, in accordance with accepted academic practice. No use, distribution or reproduction is permitted which does not comply with these terms. 